Korean J. Math. 21 (2013), No. 2, pp. 125-150

http://dx.doi.org/10.11568/kjm.2013.21.2.125

\title{
ERROR ESTIMATES FOR THE FULLY DISCRETE STABILIZED GAUGE-UZAWA METHOD PART I: THE NAVIER-STOKES EQUATIONS
}

\author{
JAE-Hong PyO
}

\begin{abstract}
The stabilized Gauge-Uzawa method (SGUM), which is a second order projection type algorithm to solve the time-dependent Navier-Stokes equations, has been newly constructed in 2013 Pyo's paper. The accuracy of SGUM has been proved only for time discrete scheme in the same paper, but it is crucial to study for fully discrete scheme, because the numerical errors depend on discretizations for both space and time, and because discrete spaces between velocity and pressure can not be chosen arbitrary. In this paper, we find out properties of the fully discrete SGUM and estimate its errors and stability to solve the evolution Navier-Stokes equations. The main difficulty in this estimation arises from losing some cancellation laws due to failing divergence free condition of the discrete velocity function. This result will be extended to Boussinesq equations in the continuous research (part II) and is essential in the study of part II.
\end{abstract}

Received March 13, 2013. Revised May 28, 2013. Accepted June 5, 2013.

2010 Mathematics Subject Classification: 65M12, 65M15, 76D05.

Key words and phrases: Projection method, Gauge-Uzawa method, the rotational form of pressure correction method, Navier-Stokes equation, incompressible fluids.

This study was supported by 2013 Research Grant from Kangwon National University (No. 120131425).

(c) The Kangwon-Kyungki Mathematical Society, 2013.

This is an Open Access article distributed under the terms of the Creative commons Attribution Non-Commercial License (http://creativecommons.org/licenses/by -nc/3.0/) which permits unrestricted non-commercial use, distribution and reproduction in any medium, provided the original work is properly cited. 


\section{Introduction}

Given an open bounded polyhedral domain $\Omega$ in $\mathbb{R}^{d}$, with $d=2$ or 3 , we consider the time-dependent Navier-Stokes equations of incompressible fluids:

$$
\begin{aligned}
\mathbf{u}_{t}+(\mathbf{u} \cdot \nabla) \mathbf{u}+\nabla p-\mu \triangle \mathbf{u} & =\mathbf{f}, & & \text { in } \Omega, \\
\nabla \cdot \mathbf{u} & =0, & & \text { in } \Omega, \\
\mathbf{u}(0, \mathbf{x}) & =\mathbf{u}^{0}, & & \text { in } \Omega,
\end{aligned}
$$

with vanishing Dirichlet boundary condition $\mathbf{u}=\mathbf{0}$ on $\partial \Omega$ and pressure mean-value $\int_{\Omega} p=0$. The primitive variables are the (vector) velocity $\mathbf{u}$ and the (scalar) pressure $p$. The viscosity $\mu=R e^{-1}$ is the reciprocal of the Reynolds number Re.

The most popular solvers of (1.1) are the projection type methods which were introduced independently by Chorin [3] and Temam [17] in the late 60 's to decouple $\mathbf{u}$ and $p$ and thus reduce the computational cost. And the methods quickly gained popularity in the computational fluid dynamics community, and over the years, an enormous amount of efforts have been devoted to develop more accurate and efficient projection type schemes. One branch of the projection type methods is the Gauge-Uzawa method (GUM) which has been constructed in [11] to solve (1.1). GUM enhanced to solve more complicated problems which are the Boussinesq equations in [12] and the non-constant density fluid problems in [16]. However, GUM has been studied only for the first order backward Euler time scheme except normal mode error analysis for the Stokes equations in [15]. We construct second order GUM and analyze the superiority of the method in normal mode space in [15]. We also discover that the method is equivalent in continuous level to the consistent splitting method which is studied in [6]. But we could not obtain any theoretical proofs for them via energy estimate even for stability condition. Because we found out weak stability performance through numerical tests, we concentrate on overcoming the weak stability constraint without losing advantages of GUM and then we obtain the stabilized Gauge-Uzawa method (SGUM) which is based on the second order backward Euler time discrete scheme in [14]. And we estimated errors for the Navier Stokes equations via energy error estimate, but the error evaluation carried out only for time discrete scheme. The study for time is not enough to guarantee accuracy, because numerical errors depend on both time and 
space discretizations and because discrete spaces between velocity and pressure can not be chosen arbitrary (see Assumption 3 below). We can find an example in [13]: the numerical tests of the Van Kan method does not display second order convergence in [13], even though the optimal accuracy for the method had been proved for the time discrete scheme. Thus it is crucial to verify error decay for fully discrete algorithm and so we will estimate optimal convergence and stability for the fully discrete SGUM in this paper.

In the other direction, the rotational form of pressure-correction method has been constructed in [19]. The errors of the method have been estimated via energy estimate in [7] and via normal mode analysis in [15]. But both proofs had been carried out only for Stokes equations. In [14], they discover that SGUM is an equivalent method to the rotational form of pressure-correction method in the view of semi-discrete level. The main difference of two methods is pressure expression: the pressure in the rotational form of pressure-correction method is designed to be updated from previous step value $\left(p^{n+1}=p^{n}+\cdots\right)$ in contrast to SGUM. This pressure expression acts upon main obstacle to treat convection term, and so the study in [7] had limited only for Stokes equations.

In this paper, we will prove stability and estimate errors for the fully discrete SGUM which is stated in Algorithm 1 to solve Navier Stokes equations. The main difficulty in this estimation arise from losing some cancellation laws due to failing divergence free condition of the discrete velocity function. In order to overcome this deficit, we introduce discontinuous velocity to hold (1.8) in the fully discrete Algorithm 1. The result in this paper will be extended to Boussinesq equations in the continuous research (part II) and is essential in the study of part II. We will denote $\tau$ as the time marching size. Also we will use $\delta$ as difference of two consecutive functions, for example, for any sequence function $z^{n+1}$,

$$
\delta z^{n+1}=z^{n+1}-z^{n}, \quad \delta \delta z^{n+1}=\delta\left(\delta z^{n+1}\right)=z^{n+1}-2 z^{n}+z^{n-1}, \quad \cdots .
$$

In order to introduce the finite element discretizations we need further notations. Let $H^{s}(\Omega)$ be the Sobolev space with $s$ derivatives in $L^{2}(\Omega)$, set $\mathbf{L}^{2}(\Omega)=\left(L^{2}(\Omega)\right)^{d}$ and $\mathbf{H}^{s}(\Omega)=\left(H^{s}(\Omega)\right)^{d}$, where $d=2$ or 3 , and denote by $L_{0}^{2}(\Omega)$ the subspace of $L^{2}(\Omega)$ of functions with vanishing meanvalue. We indicate with $\|\cdot\|_{s}$ the norm in $H^{s}(\Omega)$, and with $\langle\cdot, \cdot\rangle$ the inner product in $L^{2}(\Omega)$. Let $\mathfrak{T}=\{K\}$ be a shape-regular quasi-uniform partition of $\Omega$ of meshsize $h$ into closed elements $K[1,2,5]$. The vector 
and scalar finite element spaces are:

$$
\begin{aligned}
\mathbb{W}_{h} & :=\left\{\mathbf{w}_{h} \in \mathbf{L}^{2}(\Omega):\left.\mathbf{w}_{h}\right|_{K} \in \mathcal{P}(K) \quad \forall K \in \mathfrak{T}\right\}, \quad \mathbb{V}_{h}:=\mathbb{W}_{h} \cap \mathbf{H}_{0}^{1}(\Omega), \\
\mathbb{P}_{h} & :=\left\{q_{h} \in L_{0}^{2}(\Omega) \cap C^{0}(\Omega):\left.q_{h}\right|_{K} \in \mathcal{Q}(K) \quad \forall K \in \mathfrak{T}\right\},
\end{aligned}
$$

where $\mathcal{P}(K)$ and $\mathcal{Q}(K)$ are spaces of polynomials with degree bounded uniformly with respect to $K \in \mathfrak{T}[2,5]$. We stress that the space $\mathbb{P}_{h}$ is composed of continuous functions to make sense. This implies the crucial equality

$$
\left\langle\nabla \cdot \mathbf{w}_{h}, s_{h}\right\rangle=-\left\langle\mathbf{w}_{h}, \nabla s_{h}\right\rangle, \quad \forall \mathbf{w}_{h} \in \mathbb{V}_{h}, \forall s_{h} \in \mathbb{P}_{h} .
$$

Using the following discrete counterpart of the form

$$
\begin{gathered}
\mathcal{N}(\mathbf{u}, \mathbf{v}, \mathbf{w}):=\langle(\mathbf{u} \cdot \nabla) \mathbf{v}, \mathbf{w}\rangle \\
\mathcal{N}\left(\mathbf{u}_{h}, \mathbf{v}_{h}, \mathbf{w}_{h}\right):=\frac{1}{2}\left\langle\left(\mathbf{u}_{h} \cdot \nabla\right) \mathbf{v}_{h}, \mathbf{w}_{h}\right\rangle-\frac{1}{2}\left\langle\left(\mathbf{u}_{h} \cdot \nabla\right) \mathbf{w}_{h}, \mathbf{v}_{h}\right\rangle,
\end{gathered}
$$

We now introduce the fully discrete stabilized Gauge-Uzawa method (SGUM) which is studied for semi-discrete level in [14].

Algorithm 1 (The fully discrete stabilized Gauge-Uzawa method). Compute $\mathbf{u}_{h}^{1}$ and $p_{h}^{1}$ via any first order projection method and set $\psi_{h}^{1}=$ $\frac{-2 \tau}{3} p_{h}^{1}$ and $q_{h}^{1}=0$. Repeat for $1 \leq n \leq N=\left[\frac{T}{\tau}-1\right]$.

Step 1: Set $\mathbf{u}_{h}^{*}=2 \mathbf{u}_{h}^{n}-\mathbf{u}_{h}^{n-1}$ and find $\widehat{\mathbf{u}}_{h}^{n+1} \in \mathbb{V}_{h}$ as the solution of

$$
\begin{array}{r}
\frac{1}{2 \tau}\left\langle 3 \widehat{\mathbf{u}}_{h}^{n+1}-4 \mathbf{u}_{h}^{n}+\mathbf{u}_{h}^{n-1}, \mathbf{w}_{h}\right\rangle+\left\langle\nabla p_{h}^{n}, \mathbf{w}_{h}\right\rangle+\mathcal{N}\left(\mathbf{u}_{h}^{*}, \widehat{\mathbf{u}}_{h}^{n+1}, \mathbf{w}_{h}\right) \\
+\mu\left\langle\nabla \widehat{\mathbf{u}}_{h}^{n+1}, \nabla \mathbf{w}_{h}\right\rangle=\left\langle\mathbf{f}\left(t^{n+1}\right), \mathbf{w}_{h}\right\rangle, \quad \forall \mathbf{w}_{h} \in \mathbb{V}_{h} .
\end{array}
$$

Step 2: Find $\psi_{h}^{n+1} \in \mathbb{P}_{h}$ as the solution of

$$
\left\langle\nabla \psi_{h}^{n+1}, \nabla \phi_{h}\right\rangle=\left\langle\nabla \psi_{h}^{n}, \nabla \phi_{h}\right\rangle+\left\langle\nabla \cdot \widehat{\mathbf{u}}_{h}^{n+1}, \phi_{h}\right\rangle, \quad \forall \phi_{h} \in \mathbb{P}_{h} .
$$

Step 3: Update $\mathbf{u}_{h}^{n+1}$ and $q_{h}^{n+1} \in \mathbb{P}_{h}$ according to

$$
\begin{gathered}
\mathbf{u}_{h}^{n+1}=\widehat{\mathbf{u}}_{h}^{n+1}+\nabla\left(\psi_{h}^{n+1}-\psi_{h}^{n}\right), \\
\left\langle q_{h}^{n+1}, \phi_{h}\right\rangle=\left\langle q_{h}^{n}, \phi_{h}\right\rangle-\left\langle\nabla \cdot \widehat{\mathbf{u}}_{h}^{n+1}, \phi_{h}\right\rangle, \quad \forall \phi_{h} \in \mathbb{P}_{h} .
\end{gathered}
$$

Step 4: Update pressure $p_{h}^{n+1}$ by

$$
p_{h}^{n+1}=-\frac{3 \psi_{h}^{n+1}}{2 \tau}+\mu q_{h}^{n+1} .
$$


REMARK 1.1 (Discontinuity of $\mathbf{u}_{h}^{n+1}$ ). We note that $\mathbf{u}_{h}^{n+1}$ is a discontinuous function across inter-element boundaries and that, in light of (1.4) and (1.5), $\mathbf{u}_{h}^{n+1}$ is discrete divergence free in the sense that

$$
\left\langle\nabla \cdot \mathbf{u}_{h}^{n+1}, \phi_{h}\right\rangle=\left\langle\mathbf{u}_{h}^{n+1}, \nabla \phi_{h}\right\rangle=0, \quad \forall \phi_{h} \in \mathbb{P}_{h} .
$$

We now summarize the results of this paper along with organization. We introduce appropriate Assumptions 1-5 in $\S 2$ and introduce well known lemmas. In $\S 3$, we prove stability.

ThEOREM 1 (Stability). The SGUM is unconditionally stable in the sense that, for all $\tau>0$, the following a priori bound holds:

$$
\begin{aligned}
& \left\|\mathbf{u}_{h}^{N+1}\right\|_{0}^{2}+\left\|\widehat{\mathbf{u}}_{h}^{N+1}\right\|_{0}^{2}+\left\|2 \mathbf{u}_{h}^{N+1}-\mathbf{u}_{h}^{N}\right\|_{0}^{2}+3\left\|\nabla \psi_{h}^{N+1}\right\|_{0}^{2}+2 \mu \tau\left\|q_{h}^{N+1}\right\|_{0}^{2} \\
& +\sum_{n=1}^{N}\left(\left\|\delta \delta \mathbf{u}_{h}^{n+1}\right\|_{0}^{2}+3\left\|\nabla \delta \psi_{h}^{n+1}\right\|_{0}^{2}\right)+\mu \tau \sum_{n=1}^{N}\left\|\nabla \widehat{\mathbf{u}}_{h}^{n+1}\right\|_{0}^{2} \\
& \leq\left\|\mathbf{u}_{h}^{1}\right\|_{0}^{2}+\left\|2 \mathbf{u}_{h}^{1}-\mathbf{u}_{h}^{0}\right\|_{0}^{2}+3\left\|\nabla \psi_{h}^{1}\right\|_{0}^{2}+2 \mu \tau\left\|q_{h}^{1}\right\|_{0}^{2}+C \tau \sum_{n=1}^{N}\left\|\mathbf{f}\left(t^{n+1}\right)\right\|_{-1}^{2} .
\end{aligned}
$$

We then prove the following accuracy results through several lemmas in $\S 4$.

TheOREM 2 (Error estimates). Suppose the exact solution of (1.1) is smooth enough and $\tau=C h$. If Assumptions 1-5 below hold, then the errors of Algorithm 1 are bounded by

$$
\begin{aligned}
& \tau \sum_{n=1}^{N}\left(\left\|\mathbf{u}\left(t^{n+1}\right)-\mathbf{u}_{h}^{n+1}\right\|_{0}^{2}+\left\|\mathbf{u}\left(t^{n+1}\right)-\widehat{\mathbf{u}}_{h}^{n+1}\right\|_{0}^{2}\right) \leq C\left(\tau^{4}+h^{4}\right), \\
& \tau \sum_{n=1}^{N}\left(\left\|\mathbf{u}\left(t^{n+1}\right)-\widehat{\mathbf{u}}_{h}^{n+1}\right\|_{1}^{2}+\left\|p\left(t^{n+1}\right)-p_{h}^{n+1}\right\|_{0}^{2}\right) \leq C\left(\tau^{2}+h^{2}\right) .
\end{aligned}
$$

We finally conclude in $\S 5$ with numerical experiments.

\section{Preliminaries}

In this section, we introduce 5 assumptions and well known lemmas to use in proofs of main theorems. We resort to a duality argument via 
the following Stokes equations:

$$
\begin{aligned}
-\triangle \mathbf{v}+\nabla r & =\mathbf{g}, & & \text { in } \Omega, \\
\nabla \cdot \mathbf{v} & =0, & & \text { in } \Omega,
\end{aligned}
$$

with Dirichlet boundary condition $\mathbf{v}=0$ on $\partial \Omega$. We now state a basic assumption about $\Omega$.

Assumption 1 (Regularity of $\Omega$ ). The unique solution $\{\mathbf{v}, r\}$ of the steady Stokes equations (2.1) satisfies

$$
\|\mathbf{v}\|_{2}+\|r\|_{1} \leq C\|\mathbf{g}\|_{0} .
$$

We remark that the validity of Assumption 1 is known if $\partial \Omega$ is of class $\mathbf{C}^{2}$ $[4,8]$, or if $\partial \Omega$ is a two-dimensional convex polygon [10], and is generally believed for convex polyhedral [8].

In order to launch Algorithm 1, we need to set $\left(\mathbf{u}_{h}^{1}, p_{h}^{1}\right)$ via any first order projection method which holds the following condition.

Assumption 2 (Initial setting). Let $\left(\mathbf{u}\left(t^{1}\right), p\left(t^{1}\right)\right)$ be the exact solution of (1.1) at $t=t^{1}$. The initial value $\left(\mathbf{u}_{h}^{1}, p_{h}^{1}\right)$ satisfies

$$
\begin{aligned}
& \left\|\mathbf{u}\left(t^{1}\right)-\mathbf{u}_{h}^{1}\right\|_{0} \leq C\left(\tau^{2}+h^{2}\right), \\
& \left\|\mathbf{u}\left(t^{1}\right)-\mathbf{u}_{h}^{1}\right\|_{1}+\left\|p\left(t^{1}\right)-p_{h}^{1}\right\|_{1} \leq C(\tau+h) .
\end{aligned}
$$

We impose the following properties for relations between the spaces $\mathbb{V}_{h}$ and $\mathbb{P}_{h}$.

Assumption 3 (Discrete Inf-Sup condition). There exists a constant $\beta>0$ such that

$$
\inf _{s_{h} \in \mathbb{P}_{h}} \sup _{\mathbf{w}_{h} \in \mathbb{V}_{h}} \frac{\left\langle\nabla \cdot \mathbf{w}_{h}, s_{h}\right\rangle}{\left\|\mathbf{w}_{h}\right\|_{1}\left\|s_{h}\right\|_{0}} \geq \beta .
$$

Assumption 4 (Shape regularity and quasiuniformity $[1,2,5]$ ). There exists a constant $C>0$ such that the ratio between the diameter $h_{K}$ of an element $K \in \mathfrak{T}$ and the diameter of the largest ball contained in $K$ is bounded uniformly by $C$, and $h_{K}$ is comparable with the meshsize $h$ for all $K \in \mathfrak{T}$.

Assumption 5 (Approximability $[1,2,5]$ ). For each $(\mathbf{w}, s) \in \mathbf{H}^{2}(\Omega) \times$ $H^{1}(\Omega)$, there exist approximations $\left(\mathbf{w}_{h}, s_{h}\right) \in \mathbb{V}_{h} \times \mathbb{P}_{h}$ such that

$\left\|\mathbf{w}-\mathbf{w}_{h}\right\|_{0}+h\left\|\mathbf{w}-\mathbf{w}_{h}\right\|_{1} \leq C h^{2}\|\mathbf{w}\|_{2} \quad$ and $\quad\left\|s-s_{h}\right\|_{0} \leq C h\|s\|_{1}$.

The following elementary but crucial relations are derived in [18]. 
LEMma 2.1 (Inverse inequality). If $I_{h}$ denotes the Clement interpolant, then

$$
\left\|I_{h} \mathbf{w}\right\|_{\mathbf{L}^{3}(\Omega)} \leq C h^{-d / 6}\left\|I_{h} \mathbf{w}\right\|_{0}, \quad \text { and } \quad\left\|\mathbf{w}-I_{h} \mathbf{w}\right\|_{\mathbf{L}^{3}(\Omega)} \leq C h^{2-d / 6}\|\mathbf{w}\|_{2} .
$$

Lemma 2.2 (Div-grad relation). If $\mathbf{w} \in \mathbf{H}_{0}^{1}(\Omega)$, then

$$
\|\nabla \cdot \mathbf{w}\|_{0} \leq\|\nabla \mathbf{w}\|_{0} .
$$

Let now $\left(\mathbf{v}_{h}, r_{h}\right) \in \mathbb{V}_{h} \times \mathbb{P}_{h}$ indicate the finite element solution of (2.1), namely,

$$
\begin{aligned}
\left\langle\nabla \mathbf{v}_{h}, \nabla \mathbf{w}_{h}\right\rangle-\left\langle r_{h}, \nabla \cdot \mathbf{w}_{h}\right\rangle & =\left\langle\mathbf{g}, \mathbf{w}_{h}\right\rangle, & & \forall \mathbf{w}_{h} \in \mathbb{V}_{h}, \\
\left\langle\nabla \cdot \mathbf{w}_{h}, s_{h}\right\rangle & =0, & & \forall s_{h} \in \mathbb{P}_{h} .
\end{aligned}
$$

Then we can find the well known lemmas in $[1,2,5]$ :

LEMma 2.3 (Error estimates for mixed FEM). Let $(\mathbf{v}, s) \in \mathbf{H}_{0}^{1}(\Omega) \times$ $L_{0}^{2}(\Omega)$ be the solutions of $(2.1)$ and $\left(\mathbf{v}_{h}, s_{h}\right)=\mathfrak{S}_{h}(\mathbf{v}, s) \in \mathbb{V}_{h} \times \mathbb{P}_{h}$ be the Stokes projections defined by (2.2), respectively. If Assumptions 1 and 3-5 hold, then

$$
\begin{gathered}
\left\|\mathbf{v}-\mathbf{v}_{h}\right\|_{0}+h\left\|\mathbf{v}-\mathbf{v}_{h}\right\|_{1}+h\left\|r-r_{h}\right\|_{0} \leq C h^{2}\left(\|\mathbf{v}\|_{2}+\|r\|_{1}\right), \\
\left\|\mathbf{v}-\mathbf{v}_{h}\right\|:=\left\|\mathbf{v}-\mathbf{v}_{h}\right\|_{\mathbf{L}^{\infty}(\Omega)}+\left\|\nabla\left(\mathbf{v}-\mathbf{v}_{h}\right)\right\|_{\mathbf{L}^{3}(\Omega)} \leq C\|\mathbf{g}\|_{0} .
\end{gathered}
$$

Proof. Inequality (2.3) is standard $[1,2,5]$. To establish (2.4), we just deal with the $\mathbf{L}^{\infty}$-norm since the other can be treated similarly. If $I_{h}$ denotes the Clement interpolant, then $\left\|\mathbf{v}-I_{h} \mathbf{v}\right\|_{\mathbf{L}^{\infty}(\Omega)} \leq C\|\mathbf{v}\|_{2}$ and

$$
\left\|I_{h} \mathbf{v}-\mathbf{v}_{h}\right\|_{\mathbf{L}^{\infty}(\Omega)} \leq C h^{-d / 2}\left\|I_{h} \mathbf{v}-\mathbf{v}_{h}\right\|_{\mathbf{L}^{2}(\Omega)} \leq C\|\mathbf{v}\|_{2}
$$

as a consequence of an inverse estimate and (2.3). This completes the proof.

REMARK $2.4\left(H^{1}\right.$ stability of $\left.r_{h}\right)$. The bound $\left\|\nabla r_{h}\right\|_{0} \leq C\left(\|\mathbf{v}\|_{2}+\|r\|_{1}\right)$ is a simple by-product of (2.3). To see this, we add and subtract $I_{h} r$, use the stability of $I_{h}$ in $H^{1}$, and observe that (2.3) implies $\left\|\nabla\left(r_{h}-I_{h} r\right)\right\|_{0} \leq$ $C h^{-1}\left\|r_{h}-I_{h} r\right\|_{0} \leq C$.

We finally state without proof several properties of the nonlinear form $\mathcal{N}$. In view of (1.2), we have a following properties of $\mathcal{N}$ for all $\mathbf{u}_{h}, \mathbf{v}_{h}, \mathbf{w}_{h} \in \mathbb{V}_{h}$ :

$$
\mathcal{N}\left(\mathbf{u}_{h}, \mathbf{v}_{h}, \mathbf{w}_{h}\right)=-\mathcal{N}\left(\mathbf{u}_{h}, \mathbf{w}_{h}, \mathbf{v}_{h}\right), \quad \mathcal{N}\left(\mathbf{u}_{h}, \mathbf{v}_{h}, \mathbf{v}_{h}\right)=0,
$$


and

$\nabla \cdot \mathbf{u}=0 \Rightarrow \mathcal{N}\left(\mathbf{u}, \mathbf{v}_{h}, \mathbf{w}_{h}\right)=\left\langle(\mathbf{u} \cdot \nabla) \mathbf{v}_{h}, \mathbf{w}_{h}\right\rangle=-\left\langle(\mathbf{u} \cdot \nabla) \mathbf{w}_{h}, \mathbf{v}_{h}\right\rangle$.

Applying Sobolev imbedding Lemma yields the following useful results.

LEMmA 2.5 (Bounds on nonlinear convection $[8,11]$ ). Let $\mathbf{u}, \mathbf{v} \in \mathbf{H}^{2}(\Omega)$ with $\nabla \cdot \mathbf{u}=0$, and let $\mathbf{u}_{h}, \mathbf{v}_{h}, \mathbf{w}_{h} \in \mathbb{V}_{h}$. Then

$$
\begin{gathered}
\mathcal{N}\left(\mathbf{u}, \mathbf{v}_{h}, \mathbf{w}_{h}\right) \leq C\left\{\begin{array}{l}
\|\mathbf{u}\|_{1}\left\|\mathbf{v}_{h}\right\|_{1}\left\|\mathbf{w}_{h}\right\|_{1} \\
\|\mathbf{u}\|_{2}\left\|\mathbf{v}_{h}\right\|_{1}\left\|\mathbf{w}_{h}\right\|_{0} \\
\|\mathbf{u}\|_{2}\left\|\mathbf{v}_{h}\right\|_{0}\left\|\mathbf{w}_{h}\right\|_{1}
\end{array}\right. \\
\mathcal{N}\left(\mathbf{u}_{h}, \mathbf{v}, \mathbf{w}_{h}\right) \leq C\left\|\mathbf{u}_{h}\right\|_{0}\|\mathbf{v}\|_{2}\left\|\mathbf{w}_{h}\right\|_{1} .
\end{gathered}
$$

In addition

$$
\mathcal{N}\left(\mathbf{u}_{h}, \mathbf{v}_{h}, \mathbf{w}_{h}\right) \leq C\left\{\begin{array}{l}
\left\|\mathbf{u}_{h}\right\|_{0}\left\|\mathbf{v}_{h}\right\|\left\|\mathbf{w}_{h}\right\|_{1} \\
\left\|\mathbf{u}_{h}\right\|_{\mathbf{L}^{3}(\Omega)}\left\|\mathbf{v}_{h}\right\|_{1}\left\|\mathbf{w}_{h}\right\|_{1} .
\end{array}\right.
$$

We will use the following algebraic identities frequently to treat time derivative terms.

LEMMA 2.6 (Inner product of time derivative terms). For any sequence $\left\{z^{n}\right\}_{n=0}^{N}$, we have

$$
\begin{gathered}
2\left\langle 3 z^{n+1}-4 z^{n}+z^{n-1}, z^{n+1}\right\rangle \\
=\delta\left\|z^{n+1}\right\|_{0}^{2}+\delta\left\|2 z^{n+1}-z^{n}\right\|_{0}^{2}+\left\|\delta \delta z^{n+1}\right\|_{0}^{2}, \\
2\left\langle z^{n+1}-z^{n}, z^{n+1}\right\rangle=\left\|z^{n+1}\right\|_{0}^{2}-\left\|z^{n}\right\|_{0}^{2}+\left\|z^{n+1}-z^{n}\right\|_{0}^{2},
\end{gathered}
$$

and

$$
2\left\langle z^{n+1}-z^{n}, z^{n}\right\rangle=\left\|z^{n+1}\right\|_{0}^{2}-\left\|z^{n}\right\|_{0}^{2}-\left\|z^{n+1}-z^{n}\right\|_{0}^{2} .
$$

\section{Proof of stability}

In this section, we prove Theorem 1 . We start the proof with rewriting the momentum equation (1.3) by using (1.5) and (1.7) as follows:

$$
\begin{aligned}
& \frac{1}{2 \tau}\left\langle 3 \mathbf{u}_{h}^{n+1}-4 \mathbf{u}_{h}^{n}+\mathbf{u}_{h}^{n-1}, \mathbf{w}_{h}\right\rangle+\mathcal{N}\left(2 \mathbf{u}_{h}^{n}-\mathbf{u}_{h}^{n-1}, \widehat{\mathbf{u}}_{h}^{n+1}, \mathbf{w}_{h}\right) \\
& -\left\langle\nabla\left(\frac{3}{2 \tau} \psi_{h}^{n+1}-\mu q_{h}^{n}\right), \mathbf{w}_{h}\right\rangle+\mu\left\langle\nabla \widehat{\mathbf{u}}_{h}^{n+1}, \nabla \mathbf{w}_{h}\right\rangle=\left\langle\mathbf{f}\left(t^{n+1}\right), \mathbf{w}_{h}\right\rangle .
\end{aligned}
$$


We now choose $\mathbf{w}_{h}=4 \tau \widehat{\mathbf{u}}_{h}^{n+1}$ and use (2.9) to get

$$
\begin{aligned}
& \delta\left\|\mathbf{u}_{h}^{n+1}\right\|_{0}^{2}+\delta\left\|2 \mathbf{u}_{h}^{n+1}-\mathbf{u}_{h}^{n}\right\|_{0}^{2}+\left\|\delta \delta \mathbf{u}_{h}^{n+1}\right\|_{0}^{2}+4 \tau \mu\left\|\nabla \widehat{\mathbf{u}}_{h}^{n+1}\right\|_{0}^{2} \\
& =6\left\langle\nabla \psi_{h}^{n+1}, \widehat{\mathbf{u}}_{h}^{n+1}\right\rangle+4 \tau \mu\left\langle q_{h}^{n}, \nabla \cdot \widehat{\mathbf{u}}_{h}^{n+1}\right\rangle+4 \tau\left\langle\mathbf{f}\left(t^{n+1}\right), \widehat{\mathbf{u}}_{h}^{n+1}\right\rangle,
\end{aligned}
$$

and we denote by $A_{i}$, for $i=1,2,3$, the three terms in the right hand side. We note here that convection term is vanished by (2.5). In conjunction with $\widehat{\mathbf{u}}_{h}^{n+1}=\mathbf{u}_{h}^{n+1}-\nabla \delta \psi_{h}^{n+1},(2.10)$ yields

$$
\begin{aligned}
A_{1} & =-6\left\langle\nabla \psi_{h}^{n+1}, \nabla \delta \psi_{h}^{n+1}\right\rangle \\
& =-3\left(\left\|\nabla \psi_{h}^{n+1}\right\|_{0}^{2}-\left\|\nabla \psi_{h}^{n}\right\|_{0}^{2}+\left\|\nabla \delta \psi_{h}^{n+1}\right\|_{0}^{2}\right) .
\end{aligned}
$$

Before we estimate $A_{2}$, we evaluate an inequality via choosing $\phi_{h}=\delta q_{h}^{n+1}$ in (1.6) to get

$$
\left\|\delta q_{h}^{n+1}\right\|_{0}^{2}=-\left\langle\nabla \cdot \widehat{\mathbf{u}}_{h}^{n+1}, \delta q_{h}^{n+1}\right\rangle \leq\left\|\nabla \cdot \widehat{\mathbf{u}}_{h}^{n+1}\right\|_{0}\left\|\delta q_{h}^{n+1}\right\|_{0} .
$$

In the view of Lemma 2.2, we conclude $\left\|\delta q_{h}^{n+1}\right\|_{0}^{2} \leq\left\|\nabla \cdot \widehat{\mathbf{u}}_{h}^{n+1}\right\|_{0}^{2} \leq$ $\left\|\nabla \widehat{\mathbf{u}}_{h}^{n+1}\right\|_{0}^{2}$, whence

$$
\begin{aligned}
A_{2}= & -4 \mu \tau\left\langle q_{h}^{n}, \delta q_{h}^{n+1}\right\rangle=-2 \mu \tau\left(\left\|q_{h}^{n+1}\right\|_{0}^{2}-\left\|q_{h}^{n}\right\|_{0}^{2}-\left\|\delta q_{h}^{n+1}\right\|_{0}^{2}\right) \\
& \leq-2 \mu \tau\left(\left\|q_{h}^{n+1}\right\|_{0}^{2}-\left\|q_{h}^{n}\right\|_{0}^{2}\right)+2 \mu \tau\left\|\nabla \widehat{\mathbf{u}}_{h}^{n+1}\right\|_{0}^{2} .
\end{aligned}
$$

Clearly, we have

$$
A_{3} \leq C \frac{\tau}{\mu}\left\|\mathbf{f}\left(t^{n+1}\right)\right\|_{-1}^{2}+\tau \mu\left\|\nabla \widehat{\mathbf{u}}_{h}^{n+1}\right\|_{0}^{2} .
$$

Inserting $A_{1}-A_{3}$ back into (3.1) and summing over $n$ from 1 to $N$ lead (1.9) by help of $\left\|\widehat{\mathbf{u}}_{h}^{n+1}\right\|_{0}^{2}=\left\|\mathbf{u}_{h}^{n+1}\right\|_{0}^{2}+\left\|\nabla \delta \psi_{h}^{n+1}\right\|_{0}^{2}$ which comes form (1.8).

\section{Error estimates}

In this section, we prove Theorem 2 which is error estimates for SGUM of Algorithm 1. This proof is carried out through several lemmas. We start the proof with defining $\left(\mathbf{U}_{h}^{n+1}, P_{h}^{n+1}\right):=\mathfrak{S}_{h}\left(\mathbf{u}\left(t^{n+1}\right), p\left(t^{n+1}\right)\right) \in$ $\mathbb{V}_{h} \times \mathbb{P}_{h}$ to be the Stokes projection of the true solution at time $t^{n+1}$. It 
means that $\left(\mathbf{U}_{h}^{n+1}, P_{h}^{n+1}\right) \in \mathbb{V}_{h} \times \mathbb{P}_{h}$ is the solution of, for all $\mathbf{w}_{h} \in \mathbb{V}_{h}$ and for all $s_{h} \in \mathbb{P}_{h}$,

$$
\begin{aligned}
\left\langle\nabla \mathbf{U}_{h}^{n+1}, \nabla \mathbf{w}_{h}\right\rangle+\left\langle\nabla P_{h}^{n+1}, \mathbf{w}_{h}\right\rangle & =\left\langle\nabla \mathbf{u}\left(t^{n+1}\right), \nabla \mathbf{w}_{h}\right\rangle+\left\langle\nabla p\left(t^{n+1}\right), \mathbf{w}_{h}\right\rangle, \\
\left\langle\nabla \cdot \mathbf{U}_{h}^{n+1}, s_{h}\right\rangle & =0 .
\end{aligned}
$$

And we denote notations

$$
\mathbf{G}^{n+1}:=\mathbf{u}\left(t^{n+1}\right)-\mathbf{U}_{h}^{n+1}, \quad g^{n+1}:=p\left(t^{n+1}\right)-P_{h}^{n+1} .
$$

From Lemma 2.3, we can deduce

$$
\begin{aligned}
\left\|\mathbf{G}^{n+1}\right\|_{0}^{2}+h^{2}\left\|\mathbf{G}^{n+1}\right\|_{1}^{2} & +h^{2}\left\|g^{n+1}\right\|_{0}^{2} \\
& \leq C h^{4}\left(\left\|\mathbf{u}\left(t^{n+1}\right)\right\|_{2}^{2}+\left\|p\left(t^{n+1}\right)\right\|_{1}^{2}\right),
\end{aligned}
$$

and

$$
\begin{aligned}
\left\|\delta \mathbf{G}^{n+1}\right\|_{0}^{2}+h^{2}\left\|\delta \mathbf{G}^{n+1}\right\|_{1}^{2} & +h^{2}\left\|\delta g^{n+1}\right\|_{0}^{2} \\
& \leq C \tau h^{4} \int_{t^{n}}^{t^{n+1}}\left(\left\|\mathbf{u}_{t}(t)\right\|_{2}^{2}+\left\|p_{t}(t)\right\|_{1}^{2}\right) d t .
\end{aligned}
$$

In conjunction with the definition $\|\cdot\|$ in $(2.4)$, we can deduce

$$
\left\|\mathbf{G}^{n+1}\right\| \leq C \text {. }
$$

We now carry out error evaluate by comparing (4.10) below with (1.3)(1.7). We derive strong estimates of order 1 and use the result to prove weak estimates of order 2 for the errors

$$
\mathbf{E}_{h}^{n+1}:=\mathbf{U}_{h}^{n+1}-\mathbf{u}_{h}^{n+1}, \quad \widehat{\mathbf{E}}_{h}^{n+1}:=\mathbf{U}_{h}^{n+1}-\widehat{\mathbf{u}}_{h}^{n+1}, \quad e_{h}^{n+1}:=P_{h}^{n+1}-p_{h}^{n+1} .
$$

Then, in conjunction with (4.1), we can readily get of the same accuracy for the errors

$$
\mathbf{E}^{n+1}:=\mathbf{u}\left(t^{n+1}\right)-\mathbf{u}_{h}^{n+1}, \widehat{\mathbf{E}}^{n+1}:=\mathbf{u}\left(t^{n+1}\right)-\widehat{\mathbf{u}}_{h}^{n+1}, e^{n+1}:=p\left(t^{n+1}\right)-p_{h}^{n+1} .
$$

In addition, we denote

$$
\varepsilon_{h}^{n+1}:=P_{h}^{n+1}+\frac{3 \psi_{h}^{n+1}}{2 \tau} .
$$

Then we readily obtain the following crucial properties

$$
\begin{gathered}
\mathbf{G}^{n+1}=\mathbf{E}^{n+1}-\mathbf{E}_{h}^{n+1}=\widehat{\mathbf{E}}^{n+1}-\widehat{\mathbf{E}}_{h}^{n+1} \text { and } \widehat{\mathbf{E}}_{h}^{n+1}=\mathbf{E}_{h}^{n+1}+\nabla \delta \psi_{h}^{n+1}, \\
\widehat{\mathbf{E}}^{n+1}=\widehat{\mathbf{E}}_{h}^{n+1}=\mathbf{G}^{n+1}=\mathbf{0}, \text { on } \partial \Omega,
\end{gathered}
$$


as well as, from (1.8),

$$
\left\langle\mathbf{E}_{h}^{n+1}, \nabla \phi_{h}\right\rangle=\left\langle\mathbf{E}^{n+1}, \nabla \phi_{h}\right\rangle=\left\langle\mathbf{G}^{n+1}, \nabla \phi_{h}\right\rangle=0, \quad \forall \phi_{h} \in \mathbb{P}_{h},
$$

whence we deduce crucial orthogonality properties:

$$
\left\|\widehat{\mathbf{E}}_{h}^{n+1}\right\|_{0}^{2}=\left\|\mathbf{E}_{h}^{n+1}\right\|_{0}^{2}+\left\|\nabla \delta \psi_{h}^{n+1}\right\|_{0}^{2} .
$$

We also point out that, owing to Lemma $2.2, q_{h}^{n+1} \in \mathbb{P}_{h}$ defined in (1.6) satisfies

$$
\left\|q_{h}^{n+1}-q_{h}^{n}\right\|_{0} \leq\left\|\nabla \widehat{\mathbf{E}}^{n+1}\right\|_{0}
$$

We now estimate the first order accuracy for velocity in Lemma 4.1, and then the 2nd order accuracy for time-derivative of velocity in Lemma 4.3. The result of Lemma 4.1 is instrumental to treat convection term in proof of Lemma 4.3. We will use the results in Lemmas 4.1 and 4.3 to prove optimal error decay in Lemma 4.4. Finally, we will prove pressure error estimate in Lemma 4.5.

LEMma 4.1 (Reduced rate of convergence for velocity). Suppose the exact solution of (1.1) is smooth enough. If Assumptions 2 and 4-5 hold, then the velocity error functions satisfy

$$
\begin{aligned}
& \left\|\widehat{\mathbf{E}}_{h}^{N+1}\right\|_{0}^{2}+\left\|\mathbf{E}_{h}^{N+1}\right\|_{0}^{2}+\left\|2 \mathbf{E}_{h}^{N+1}-\mathbf{E}_{h}^{N}\right\|_{0}^{2}+\frac{1}{2}\left\|\delta \delta \mathbf{E}_{h}^{N+1}\right\|_{0}^{2}+2 \mu \tau\left\|q_{h}^{N+1}\right\|_{0}^{2} \\
& +\sum_{n=1}^{N}\left\|\nabla \delta \psi_{h}^{n+1}\right\|_{0}^{2}+\mu \tau \sum_{n=1}^{N}\left\|\nabla \widehat{\mathbf{E}}_{h}^{n+1}\right\|_{0}^{2}+\frac{4 \tau^{2}}{3}\left\|\nabla \varepsilon_{h}^{N+1}\right\|_{0}^{2} \leq C\left(\tau^{2}+h^{2}\right) .
\end{aligned}
$$

Proof. By virtue of Taylor expansion for the exact velocity $\mathbf{u}(t)$, we get

$$
\begin{array}{r}
\frac{3 \mathbf{u}\left(t^{n+1}\right)-4 \mathbf{u}\left(t^{n}\right)+\mathbf{u}\left(t^{n-1}\right)}{2 \tau}+\left(\mathbf{u}\left(t^{n+1}\right) \cdot \nabla\right) \mathbf{u}\left(t^{n+1}\right) \\
+\nabla p\left(t^{n+1}\right)-\mu \triangle \mathbf{u}\left(t^{n+1}\right)=\mathbf{R}^{n+1}+\mathbf{f}\left(t^{n+1}\right),
\end{array}
$$

where $\mathbf{R}^{n+1}:=\frac{1}{\tau} \int_{t^{n}}^{t^{n+1}} \mathbf{u}_{t t t}(s)\left(s-t^{n}\right)^{2} d s-\frac{1}{4 \tau} \int_{t^{n-1}}^{t^{n+1}} \mathbf{u}_{t t t}(s)\left(t^{n-1}-s\right)^{2} d s$ is the truncation error. In conjunction with the definition of Stokes projection $\left\{\mathbf{U}_{h}^{n+1}, P_{h}^{n+1}\right\}$, testing (4.9) with $\forall \mathbf{w}_{h} \in \mathbb{V}_{h}$ yields

$$
\begin{array}{r}
(4.10) \frac{1}{2 \tau}\left\langle 3 \mathbf{u}\left(t^{n+1}\right)-4 \mathbf{u}\left(t^{n}\right)+\mathbf{u}\left(t^{n-1}\right), \mathbf{w}_{h}\right\rangle+\left\langle\nabla P_{h}^{n+1}, \mathbf{w}_{h}\right\rangle \\
+\mu\left\langle\nabla \mathbf{U}_{h}^{n+1}, \nabla \mathbf{w}_{h}\right\rangle+\mathcal{N}\left(\mathbf{u}\left(t^{n+1}\right), \mathbf{u}\left(t^{n+1}\right), \mathbf{w}_{h}\right) \\
=\left\langle\mathbf{R}^{n+1}, \mathbf{w}_{h}\right\rangle+\left\langle\mathbf{f}\left(t^{n+1}\right), \mathbf{w}_{h}\right\rangle,
\end{array}
$$


We replace $p_{h}^{n}$ term in (1.3) by (1.7) and then subtract from (4.10) to obtain

$$
\begin{aligned}
\frac{1}{2 \tau} & \left\langle 3 \widehat{\mathbf{E}}^{n+1}-4 \mathbf{E}^{n}+\mathbf{E}^{n-1}, \mathbf{w}_{h}\right\rangle+\mu \tau\left\langle\nabla \widehat{\mathbf{E}}_{h}^{n+1}, \nabla \mathbf{w}_{h}\right\rangle \\
= & -\left\langle\nabla\left(\delta P_{h}^{n+1}+\varepsilon_{h}^{n}\right), \mathbf{w}_{h}\right\rangle+\mu\left\langle\nabla q_{h}^{n}, \mathbf{w}_{h}\right\rangle+\left\langle\mathbf{R}^{n+1}, \mathbf{w}_{h}\right\rangle \\
& +\mathcal{N}\left(2 \mathbf{u}_{h}^{n}-\mathbf{u}_{h}^{n-1}, \widehat{\mathbf{u}}_{h}^{n+1}, \mathbf{w}_{h}\right)-\mathcal{N}\left(\mathbf{u}\left(t^{n+1}\right), \mathbf{u}\left(t^{n+1}\right), \mathbf{w}_{h}\right) .
\end{aligned}
$$

Choosing $\mathbf{w}_{h}=4 \tau \widehat{\mathbf{E}}_{h}^{n+1}=4 \tau\left(\widehat{\mathbf{E}}^{n+1}-\mathbf{G}^{n+1}\right)$ in (4.11) and using (2.9), we easily get

$$
\begin{gathered}
\left\|\mathbf{E}_{h}^{n+1}\right\|_{0}^{2}+\left\|2 \mathbf{E}_{h}^{n+1}-\mathbf{E}_{h}^{n}\right\|_{0}^{2}+\left\|\delta \delta \mathbf{E}_{h}^{n+1}\right\|_{0}^{2}+6\left\|\nabla \delta \psi_{h}^{n+1}\right\|_{0}^{2} \\
-\left\|\mathbf{E}_{h}^{n}\right\|_{0}^{2}-\left\|2 \mathbf{E}_{h}^{n}-\mathbf{E}_{h}^{n-1}\right\|_{0}^{2}+4 \mu \tau\left\|\nabla \widehat{\mathbf{E}}_{h}^{n+1}\right\|_{0}^{2}=\sum_{n=1}^{6} A_{i}
\end{gathered}
$$

where

$$
\begin{array}{ll}
A_{1}:=-4 \tau \mathcal{N}\left(\mathbf{u}\left(t^{n+1}\right), \mathbf{u}\left(t^{n+1}\right), \widehat{\mathbf{E}}_{h}^{n+1}\right)+4 \tau \mathcal{N}\left(2 \mathbf{u}_{h}^{n}-\mathbf{u}_{h}^{n-1}, \widehat{\mathbf{u}}_{h}^{n+1}, \widehat{\mathbf{E}}_{h}^{n+1}\right), \\
A_{2}:=-2\left\langle 3 \mathbf{G}^{n+1}-4 \mathbf{G}^{n}+\mathbf{G}^{n-1}, \widehat{\mathbf{E}}_{h}^{n+1}\right\rangle, & A_{3}:=-4 \tau\left\langle\nabla \delta P_{h}^{n+1}, \widehat{\mathbf{E}}_{h}^{n+1}\right\rangle, \\
A_{4}:=-4 \tau\left\langle\nabla \varepsilon_{h}^{n}, \widehat{\mathbf{E}}_{h}^{n+1}\right\rangle, & A_{5}:=4 \mu \tau\left\langle\nabla q_{h}^{n}, \widehat{\mathbf{E}}_{h}^{n+1}\right\rangle, \\
A_{6}:=4 \tau\left\langle\mathbf{R}^{n+1}, \widehat{\mathbf{E}}_{h}^{n+1}\right\rangle . &
\end{array}
$$

We now estimate terms $A_{1}$ to $A_{6}$ separately. To tackle $A_{1}$, we first add and subtract $2 \mathbf{u}\left(t^{n}\right)-\mathbf{u}\left(t^{n-1}\right)$ to obtain

$$
\begin{aligned}
A_{1}= & -4 \tau \mathcal{N}\left(\delta \delta \mathbf{u}\left(t^{n+1}\right), \mathbf{u}\left(t^{n+1}\right), \widehat{\mathbf{E}}_{h}^{n+1}\right)-4 \tau \mathcal{N}\left(2 \mathbf{u}_{h}^{n}-\mathbf{u}_{h}^{n-1}, \widehat{\mathbf{E}}^{n+1}, \widehat{\mathbf{E}}_{h}^{n+1}\right) \\
& -4 \tau \mathcal{N}\left(2 \mathbf{E}^{n}-\mathbf{E}^{n-1}, \mathbf{u}\left(t^{n+1}\right), \widehat{\mathbf{E}}_{h}^{n+1}\right) .
\end{aligned}
$$

Because of $\mathcal{N}\left(2 \mathbf{u}_{h}^{n}-\mathbf{u}_{h}^{n-1}, \widehat{\mathbf{E}}_{h}^{n+1}, \widehat{\mathbf{E}}_{h}^{n+1}\right)=0$, which comes from (2.5), the second term of $A_{1}$ can be replaced by

$$
4 \tau \mathcal{N}\left(2 \mathbf{E}^{n}-\mathbf{E}^{n-1}-2 \mathbf{u}\left(t^{n}\right)+\mathbf{u}\left(t^{n-1}\right), \mathbf{G}^{n+1}, \widehat{\mathbf{E}}_{h}^{n+1}\right) .
$$

If we apply Lemma 2.5 , then we can readily obtain

$$
\begin{aligned}
& A_{1} \leq C \tau\left(\left\|\delta \delta \mathbf{u}\left(t^{n+1}\right)\right\|_{0}\left\|\mathbf{u}\left(t^{n+1}\right)\right\|_{2}+\left\|2 \mathbf{E}^{n}-\mathbf{E}^{n-1}\right\|_{0}\left\|\mathbf{u}\left(t^{n+1}\right)\right\|_{2}\right)\left\|\widehat{\mathbf{E}}_{h}^{n+1}\right\|_{1} \\
& +C \tau\left(\left\|2 \mathbf{E}^{n}-\mathbf{E}^{n-1}\right\|_{0}\left\|\mathbf{G}^{n+1}\right\|+\left\|2 \mathbf{u}\left(t^{n}\right)-\mathbf{u}\left(t^{n-1}\right)\right\|_{2}\left\|\mathbf{G}^{n+1}\right\|_{0}\right)\left\|\widehat{\mathbf{E}}_{h}^{n+1}\right\|_{1} .
\end{aligned}
$$


Since we have $\left\|\mathbf{u}\left(t^{n+1}\right)\right\|_{2}+\left\|\mathbf{G}^{n+1}\right\| \leq M$ according to (4.3), we arrive at

$$
\begin{aligned}
A_{1} \leq & C \tau\left(\left\|2 \mathbf{E}_{h}^{n}-\mathbf{E}_{h}^{n-1}\right\|_{0}^{2}+\left\|2 \mathbf{G}^{n}-\mathbf{G}^{n-1}\right\|_{0}^{2}+\left\|\mathbf{G}^{n+1}\right\|_{0}^{2}\right) \\
& +\mu \tau\left\|\nabla \widehat{\mathbf{E}}_{h}^{n+1}\right\|_{0}^{2}+\frac{C \tau^{4}}{\mu} \int_{t^{n-1}}^{t^{n+1}}\left\|\mathbf{u}_{t t t}(t)\right\|_{0}^{2} d t
\end{aligned}
$$

In light of (4.2), $A_{2}$ becomes

$$
A_{2} \leq C \tau\left\|\widehat{\mathbf{E}}_{h}^{n+1}\right\|_{0}^{2}+C h^{4} \int_{t^{n-1}}^{t^{n+1}}\left(\left\|\mathbf{u}_{t}(t)\right\|_{2}^{2}+\left\|p_{t}(t)\right\|_{1}^{2}\right) d t
$$

In order to estimate $A_{3}$ and $A_{4}$, we note $\left\langle\nabla \varepsilon_{h}^{n}, \mathbf{E}_{h}^{n+1}\right\rangle=0$ and $\widehat{\mathbf{E}}_{h}^{n+1}=$ $\mathbf{E}_{h}^{n+1}+\nabla \delta \psi_{h}^{n+1}$ according to (4.5) and (4.4), respectively. The we have

$$
\begin{aligned}
A_{3} & =-4 \tau\left\langle\nabla \delta P_{h}^{n+1}, \nabla \delta \psi_{h}^{n+1}\right\rangle \\
& \leq\left\|\nabla \delta \psi_{h}^{n+1}\right\|_{0}^{2}+C \tau^{3} \int_{t^{n}}^{t^{n+1}}\left(\left\|\mathbf{u}_{t}(t)\right\|_{2}^{2}+\left\|p_{t}(t)\right\|_{1}^{2}\right) d t
\end{aligned}
$$

In conjunction with the definition $\varepsilon_{h}^{n+1}=P_{h}^{n+1}+\frac{3 \psi_{h}^{n+1}}{2 \tau}, A_{4}$ can be evaluated by

$$
\begin{gathered}
A_{4}=-4 \tau\left\langle\nabla \varepsilon_{h}^{n}, \nabla \delta \psi_{h}^{n+1}\right\rangle=-\frac{8 \tau^{2}}{3}\left\langle\nabla \varepsilon_{h}^{n}, \nabla\left(\delta \varepsilon_{h}^{n+1}-\delta P_{h}^{n+1}\right)\right\rangle \\
\leq-\frac{4 \tau^{2}}{3}\left(\left\|\nabla \varepsilon_{h}^{n+1}\right\|_{0}^{2}-\left\|\nabla \varepsilon_{h}^{n}\right\|_{0}^{2}-\left\|\nabla \delta \varepsilon_{h}^{n+1}\right\|_{0}^{2}\right) \\
+C \tau^{3}\left\|\nabla \varepsilon_{h}^{n}\right\|_{0}^{2}+C \tau\left\|\nabla \delta P_{h}^{n+1}\right\|_{0}^{2} .
\end{gathered}
$$

If we now apply inequality $(a+b)^{2} \leq 4 a^{2}+\frac{4}{3} b^{2}$, then we can get

$$
\begin{aligned}
\frac{4 \tau^{2}}{3}\left\|\nabla \delta \varepsilon_{h}^{n+1}\right\|_{0}^{2} & =\frac{4 \tau^{2}}{3}\left\|\nabla \delta P_{h}^{n+1}+\frac{3}{2 \tau} \nabla \delta \psi_{h}^{n+1}\right\|_{0}^{2} \\
& \leq C \tau^{2}\left\|\nabla \delta P_{h}^{n+1}\right\|_{0}^{2}+4\left\|\nabla \delta \psi_{h}^{n+1}\right\|_{0}^{2}
\end{aligned}
$$

So we arrive at

$$
\begin{aligned}
A_{4} \leq & -\frac{4 \tau^{2}}{3}\left(\left\|\nabla \varepsilon_{h}^{n+1}\right\|_{0}^{2}-\left\|\nabla \varepsilon_{h}^{n}\right\|_{0}^{2}\right)+C \tau^{3}\left\|\nabla \varepsilon_{h}^{n}\right\|_{0}^{2} \\
& +4\left\|\nabla \delta \psi_{h}^{n+1}\right\|_{0}^{2}+C \tau^{2} \int_{t^{n-1}}^{t^{n+1}}\left(\left\|\mathbf{u}_{t}(t)\right\|_{2}^{2}+\left\|p_{t}(t)\right\|_{1}^{2}\right) d t
\end{aligned}
$$

In light of $\nabla \cdot \mathbf{u}\left(t^{n+1}\right)=0$ and (2.11), (1.6) and (4.7) yield

$$
\begin{aligned}
A_{5} & =4 \mu \tau\left\langle q_{h}^{n}, \nabla \cdot \widehat{\mathbf{u}}_{h}^{n+1}\right\rangle=-4 \mu \tau\left\langle q_{h}^{n}, \delta q_{h}^{n+1}\right\rangle \\
& =-2 \mu \tau\left(\left\|q_{h}^{n+1}\right\|_{0}^{2}-\left\|q_{h}^{n}\right\|_{0}^{2}-\left\|\delta q_{h}^{n+1}\right\|_{0}^{2}\right) \\
& \leq-2 \mu \tau\left(\left\|q_{h}^{n+1}\right\|_{0}^{2}-\left\|q_{h}^{n}\right\|_{0}^{2}\right)+2 \mu \tau\left\|\nabla \widehat{\mathbf{E}}_{h}^{n+1}\right\|_{0}^{2}
\end{aligned}
$$


Also we readily get

$$
A_{6} \leq C \tau\left\|\widehat{\mathbf{E}}_{h}^{n+1}\right\|_{0}^{2}+C \tau^{4} \int_{t_{n-1}}^{t^{n+1}}\left\|\mathbf{u}_{t t t}(t)\right\|_{0}^{2} d t .
$$

Replacing $A_{1}-A_{6}$ back into (4.12) and summing over $n$ from 1 to $N$ imply

$$
\begin{aligned}
& \left\|\mathbf{E}_{h}^{N+1}\right\|_{0}^{2}+\left\|2 \mathbf{E}_{h}^{N+1}-\mathbf{E}_{h}^{N}\right\|_{0}^{2}+\sum_{n=1}^{N}\left(\left\|\delta \delta \mathbf{E}_{h}^{n+1}\right\|_{0}^{2}+\left\|\nabla \delta \psi_{h}^{n+1}\right\|_{0}^{2}\right) \\
& +\frac{4 \tau^{2}}{3}\left\|\nabla \varepsilon_{h}^{N+1}\right\|_{0}^{2}+2 \mu \tau\left\|q_{h}^{N+1}\right\|_{0}^{2}+\mu \tau \sum_{n=1}^{N}\left\|\nabla \widehat{\mathbf{E}}_{h}^{n+1}\right\|_{0}^{2} \\
& \leq 2 \mu \tau\left\|q_{h}^{1}\right\|_{0}^{2}+\frac{4 \tau^{2}}{3}\left\|\nabla \varepsilon_{h}^{1}\right\|_{0}^{2}+\left\|2 \mathbf{E}^{1}-\mathbf{E}^{0}\right\|_{0}^{2}+\left\|\mathbf{E}^{1}\right\|_{0}^{2}+C \tau^{3} \sum_{n=1}^{N}\left\|\nabla \varepsilon_{h}^{n}\right\|_{0}^{2} \\
& +C \tau \sum_{n=1}^{N}\left(\left\|\widehat{\mathbf{E}}_{h}^{n+1}\right\|_{0}^{2}+\left\|2 \mathbf{E}^{n}-\mathbf{E}^{n-1}\right\|_{0}^{2}+\left\|2 \mathbf{G}^{n}-\mathbf{G}^{n-1}\right\|_{0}^{2}+\left\|\mathbf{G}^{n+1}\right\|_{0}^{2}\right) \\
& +\frac{C \tau^{4}}{\mu} \int_{t^{n-1}}^{t^{n+1}}\left\|\mathbf{u}_{t t t}(t)\right\|_{0}^{2} d t+C\left(\tau^{2}+h^{4}\right) \int_{t^{n-1}}^{t^{n+1}}\left(\left\|\mathbf{u}_{t}(t)\right\|_{2}^{2}+\left\|p_{t}(t)\right\|_{1}^{2}\right) d t .
\end{aligned}
$$

In light of $\varepsilon_{h}^{1}=P_{h}^{1}+\frac{3}{2 \tau} \psi_{h}^{1}=e_{h}^{1}$, we obtain $\left\|\nabla \varepsilon_{h}^{1}\right\|_{0}^{2} \leq C$ and the first four terms in the right hand side can be bounded by Assumption 2 and properties $\mathbf{E}^{0}=\mathbf{0}$ and $q^{1}=0$ which are directly deduced from the conditions in Algorithm 1. And the next terms can be treated by the discrete Gronwall lemma. Finally, in conjunction with (4.1) and (4.6), we arrive at (4.8) and complete this proof.

REMARK 4.2 (Suboptimal order). The suboptimal accuracy result in Lemma 4.1 is due to terms of $A_{3}$ and $A_{4}$ which come from parts of pressure in the above estimate. To improve upon this, we must get rid of the terms and so we will use duality argument in Lemma 4.4. However, this suboptimal result is essential to control convection term in proofs of next lemmas to get optimal order.

In order to use in the error estimate for time-derivative of velocity in Lemma 4.3, we need to evaluate optimal initial errors for the case $n=1$. To do this, we have to compute again (4.13) and we rewrite $A_{4}$ as

$$
\begin{aligned}
A_{4}= & -\frac{8 \tau^{2}}{3}\left\langle\nabla \varepsilon_{h}^{1}, \nabla\left(\delta \varepsilon_{h}^{2}-\delta P_{h}^{2}\right)\right\rangle \\
\leq & -\frac{4 \tau^{2}}{3}\left(\left\|\nabla \varepsilon_{h}^{2}\right\|_{0}^{2}-\left\|\nabla \varepsilon_{h}^{1}\right\|_{0}^{2}-\left\|\nabla \delta \varepsilon_{h}^{2}\right\|_{0}^{2}\right) \\
& +C \tau^{2}\left\|\nabla \varepsilon_{h}^{1}\right\|_{0}^{2}+C \tau^{2}\left\|\nabla \delta P_{h}^{n+1}\right\|_{0}^{2}
\end{aligned}
$$

and so we conclude

$$
\begin{aligned}
A_{4} \leq & -\frac{4 \tau^{2}}{3}\left\|\nabla \varepsilon_{h}^{2}\right\|_{0}^{2}+C \tau^{2}\left\|\nabla \varepsilon_{h}^{1}\right\|_{0}^{2}+4\left\|\nabla \delta \psi_{h}^{2}\right\|_{0}^{2} \\
& +C \tau^{3} \int_{t^{0}}^{t^{2}}\left(\left\|p_{t t}(t)\right\|_{1}^{2}+\left\|\mathbf{u}_{t t}(t)\right\|_{2}^{2}\right) d t
\end{aligned}
$$


In light of Assumption 2, we arrive at

$$
\begin{aligned}
\left\|\widehat{\mathbf{E}}_{h}^{2}\right\|_{0}^{2} & +\left\|\mathbf{E}_{h}^{2}\right\|_{0}^{2}+\left\|2 \mathbf{E}_{h}^{2}-\mathbf{E}_{h}^{1}\right\|_{0}^{2}+\frac{1}{2}\left\|\delta \delta \mathbf{E}_{h}^{2}\right\|_{0}^{2}+\left\|\nabla \delta \psi_{h}^{2}\right\|_{0}^{2} \\
& +\mu \tau\left\|\nabla \widehat{\mathbf{E}}_{h}^{2}\right\|_{0}^{2}+2 \mu \tau\left\|q_{h}^{2}\right\|_{0}^{2}+\frac{4 \tau^{2}}{3}\left\|\nabla \varepsilon_{h}^{2}\right\|_{0}^{2} \leq C\left(\tau^{4}+h^{4}\right) .
\end{aligned}
$$

We now start to estimate errors for time-derivative of velocity.

LEMMA 4.3 (Error estimate for time-derivative of velocity). Suppose the exact solution of (1.1) is smooth enough and $\tau=C h$. If Assumptions 2 and 4-5 hold, then the time derivative velocity error functions satisfy

$$
\begin{array}{r}
\left\|\delta \mathbf{E}_{h}^{N+1}\right\|_{0}^{2}+\left\|\delta \widehat{\mathbf{E}}_{h}^{N+1}\right\|_{0}^{2}+\left\|2 \delta \mathbf{E}_{h}^{N+1}-\delta \mathbf{E}_{h}^{N}\right\|_{0}^{2}+\frac{4 \tau^{2}}{3}\left\|\nabla \delta \varepsilon_{h}^{N+1}\right\|_{0}^{2} \\
+\sum_{n=2}^{N}\left(\left\|\delta \delta \delta \mathbf{E}_{h}^{n+1}\right\|_{0}^{2}+\left\|\nabla \delta \delta \psi_{h}^{n+1}\right\|_{0}+\mu \tau\left\|\nabla \delta \widehat{\mathbf{E}}_{h}^{n+1}\right\|_{0}^{2}\right) \\
+2 \mu \tau\left\|\delta q_{h}^{N+1}\right\|_{0}^{2} \leq C \tau^{2}\left(\tau^{2}+h^{2}\right) .
\end{array}
$$

Proof. Subtracting two consecutive formulas (4.11) and choosing by $\mathbf{w}_{h}=$ $4 \tau \delta \widehat{\mathbf{E}}_{h}^{n+1}$ yield

$$
\begin{array}{r}
\left\|\delta \mathbf{E}_{h}^{n+1}\right\|_{0}^{2}+\left\|2 \delta \mathbf{E}_{h}^{n+1}-\delta \mathbf{E}_{h}^{n}\right\|_{0}^{2}+\left\|\delta \delta \delta \mathbf{E}_{h}^{n+1}\right\|_{0}^{2}+6\left\|\nabla \delta \delta \psi_{h}^{n+1}\right\|_{0}^{2} \\
-\left\|\delta \mathbf{E}_{h}^{n}\right\|_{0}^{2}-\left\|2 \delta \mathbf{E}_{h}^{n}-\delta \mathbf{E}_{h}^{n-1}\right\|_{0}^{2}+4 \mu \tau\left\|\nabla \delta \widehat{\mathbf{E}}_{h}^{n+1}\right\|_{0}^{2}=\sum_{i=1}^{6} A_{i}
\end{array}
$$

where

$$
\begin{aligned}
& A_{1}:=-4 \tau \mathcal{N}\left(\mathbf{u}\left(t^{n+1}\right), \mathbf{u}\left(t^{n+1}\right), \delta \widehat{\mathbf{E}}_{h}^{n+1}\right)+4 \tau \mathcal{N}\left(\mathbf{u}\left(t^{n}\right), \mathbf{u}\left(t^{n}\right), \delta \widehat{\mathbf{E}}_{h}^{n+1}\right) \\
& +4 \tau \mathcal{N}\left(2 \mathbf{u}_{h}^{n}-\mathbf{u}_{h}^{n-1}, \widehat{\mathbf{u}}_{h}^{n+1}, \delta \widehat{\mathbf{E}}_{h}^{n+1}\right)-4 \tau \mathcal{N}\left(2 \mathbf{u}_{h}^{n-1}-\mathbf{u}_{h}^{n-2}, \widehat{\mathbf{u}}_{h}^{n}, \delta \widehat{\mathbf{E}}_{h}^{n+1}\right), \\
& A_{2}:=-2\left\langle 3 \delta \mathbf{G}^{n+1}-4 \delta \mathbf{G}^{n}+\delta \mathbf{G}^{n-1}, \delta \widehat{\mathbf{E}}_{h}^{n+1}\right\rangle, \\
& A_{3}:=-4 \tau\left\langle\nabla \delta \delta P_{h}^{n+1}, \delta \widehat{\mathbf{E}}_{h}^{n+1}\right\rangle, \quad A_{4}:=-4 \tau\left\langle\nabla \delta \varepsilon_{h}^{n}, \delta \widehat{\mathbf{E}}_{h}^{n+1}\right\rangle, \\
& A_{5}:=4 \mu \tau\left\langle\nabla \delta q_{h}^{n}, \delta \widehat{\mathbf{E}}_{h}^{n+1}\right\rangle, \quad A_{6}:=4 \tau\left\langle\delta \mathbf{R}^{n+1}, \delta \widehat{\mathbf{E}}_{h}^{n+1}\right\rangle .
\end{aligned}
$$

We now estimate each term $A_{1}$ to $A_{6}$ separately. We first rewrite $A_{1}$ as follows:

$$
\begin{aligned}
A_{1}= & 4 \tau \mathcal{N}\left(\delta \delta \mathbf{u}\left(t^{n}\right), \mathbf{u}\left(t^{n}\right), \delta \widehat{\mathbf{E}}_{h}^{n+1}\right)-4 \tau \mathcal{N}\left(\delta \delta \mathbf{u}\left(t^{n+1}\right), \mathbf{u}\left(t^{n+1}\right), \delta \widehat{\mathbf{E}}_{h}^{n+1}\right) \\
& +4 \tau \mathcal{N}\left(2 \mathbf{E}^{n-1}-\mathbf{E}^{n-2}, \mathbf{u}\left(t^{n}\right), \delta \widehat{\mathbf{E}}_{h}^{n+1}\right) \\
& -4 \tau \mathcal{N}\left(2 \mathbf{E}^{n}-\mathbf{E}^{n-1}, \mathbf{u}\left(t^{n+1}\right), \delta \widehat{\mathbf{E}}_{h}^{n+1}\right) \\
& +4 \tau \mathcal{N}\left(2 \mathbf{u}_{h}^{n-1}-\mathbf{u}_{h}^{n-2}, \mathbf{G}^{n}+\widehat{\mathbf{E}}_{h}^{n}, \delta \widehat{\mathbf{E}}_{h}^{n+1}\right) \\
& -4 \tau \mathcal{N}\left(2 \mathbf{u}_{h}^{n}-\mathbf{u}_{h}^{n-1}, \mathbf{G}^{n+1}+\widehat{\mathbf{E}}_{h}^{n+1}, \delta \widehat{\mathbf{E}}_{h}^{n+1}\right)
\end{aligned}
$$


and we denote by $A_{1, i}$, for $i=1,2, \cdots, 6$ the six terms in the right hand side. In estimating convection terms, we will use Lemma 2.5 frequently without notice. We recall $\|\mathbf{u}(t)\|_{2} \leq C$ to obtain

$$
\begin{aligned}
A_{1,1}+A_{1,2} & \leq C \tau\left(\left\|\delta \delta \mathbf{u}\left(t^{n+1}\right)\right\|_{0}\left\|\mathbf{u}\left(t^{n+1}\right)\right\|_{2}+\left\|\delta \delta \mathbf{u}\left(t^{n}\right)\right\|_{0}\left\|\mathbf{u}\left(t^{n}\right)\right\|_{2}\right)\left\|\delta \widehat{\mathbf{E}}_{h}^{n+1}\right\|_{1} \\
& \leq \frac{\mu \tau}{6}\left\|\nabla \delta \widehat{\mathbf{E}}_{h}^{n+1}\right\|_{0}^{2}+\frac{C \tau^{4}}{\mu} \int_{t^{n-2}}^{t^{n+1}}\left\|\mathbf{u}_{t t}(t)\right\|_{0}^{2} d t
\end{aligned}
$$

The result in Lemma 4.1, $\left\|2 \mathbf{E}^{n}-\mathbf{E}^{n-1}\right\|_{0} \leq C(\tau+h)$, is essential to treat next two convection terms. Invoking (2.7), we have

$$
\begin{gathered}
A_{1,3}+A_{1,4} \leq C \tau\left\|2 \mathbf{E}^{n}-\mathbf{E}^{n-1}\right\|_{0}\left\|\delta \mathbf{u}\left(t^{n+1}\right)\right\|_{2}\left\|\delta \widehat{\mathbf{E}}_{h}^{n+1}\right\|_{1} \\
+C \tau\left\|2 \delta \mathbf{E}^{n}-\delta \mathbf{E}^{n-1}\right\|_{0}\left\|\mathbf{u}\left(t^{n}\right)\right\|_{2}\left\|\delta \widehat{\mathbf{E}}_{h}^{n+1}\right\|_{1} \\
\leq \frac{\mu \tau}{6}\left\|\nabla \delta \widehat{\mathbf{E}}_{h}^{n+1}\right\|_{0}^{2}+\frac{C \tau}{\mu}\left\|2 \delta \mathbf{E}^{n}-\delta \mathbf{E}^{n-1}\right\|_{0}^{2}+\frac{C \tau^{2}\left(\tau^{2}+h^{2}\right)}{\mu} \int_{t_{n-1}}^{n}\left\|\mathbf{u}_{t}(t)\right\|_{2}^{2} d t .
\end{gathered}
$$

We note $\mathcal{N}\left(2 \mathbf{u}_{h}^{n}-\mathbf{u}_{h}^{n-1}, \delta \widehat{\mathbf{E}}_{h}^{n+1}, \delta \widehat{\mathbf{E}}_{h}^{n+1}\right)=0$ which comes from (2.5). Then we obtain

$$
\begin{aligned}
& A_{1,5}+A_{1,6}=-4 \tau \mathcal{N}\left(2 \delta \mathbf{u}_{h}^{n}-\delta \mathbf{u}_{h}^{n-1}, \mathbf{G}^{n+1}+\widehat{\mathbf{E}}_{h}^{n+1}, \delta \widehat{\mathbf{E}}_{h}^{n+1}\right) \\
& -4 \tau \mathcal{N}\left(2 \mathbf{u}_{h}^{n-1}-\mathbf{u}_{h}^{n-2}, \delta \mathbf{G}^{n+1}, \delta \widehat{\mathbf{E}}_{h}^{n+1}\right) \\
& =4 \tau \mathcal{N}\left(2 \delta \mathbf{E}^{n}-\delta \mathbf{E}^{n-1}-2 \delta \mathbf{u}\left(t^{n}\right)+\delta \mathbf{u}\left(t^{n-1}\right), \mathbf{G}^{n+1}+\widehat{\mathbf{E}}_{h}^{n+1}, \delta \widehat{\mathbf{E}}_{h}^{n+1}\right) \\
& +4 \tau \mathcal{N}\left(2 \mathbf{E}^{n-1}-\mathbf{E}^{n-2}-2 \mathbf{u}\left(t^{n-1}\right)+\mathbf{u}\left(t^{n-2}\right), \delta \mathbf{G}^{n+1}, \delta \widehat{\mathbf{E}}_{h}^{n+1}\right):=B_{1}+B_{2} .
\end{aligned}
$$

To attack $B_{1}$, we first note Lemma 2.1 which is, for any $\mathbf{w}_{h} \in \mathbb{V}_{h},\left\|\mathbf{w}_{h}\right\|_{\mathbf{L}^{3}(\Omega)} \leq$ $C h^{-d / 6}\left\|\mathbf{w}_{h}\right\|_{0}$.

If we apply $\left\|\widehat{\mathbf{E}}^{n+1}+\mathbf{G}^{n+1}\right\|_{0}+\sqrt{\tau+h}\left\|\widehat{\mathbf{E}}^{n+1}+\mathbf{G}^{n+1}\right\|_{1} \leq C(\tau+h)$ which is the result of Lemma 4.1, then we can conclude, in light of (2.8),

$$
\begin{aligned}
B_{1} \leq & C \tau\left\|2 \delta \mathbf{E}^{n}-\delta \mathbf{E}^{n-1}\right\|_{\mathbf{L}^{3}(\Omega)}\left\|\widehat{\mathbf{E}}_{h}^{n+1}+\mathbf{G}^{n+1}\right\|_{1}\left\|\nabla \delta \widehat{\mathbf{E}}_{h}^{n+1}\right\|_{0} \\
& +C \tau\left\|2 \delta \mathbf{u}\left(t^{n}\right)-\delta \mathbf{u}\left(t^{n-1}\right)\right\|_{2}\left\|\widehat{\mathbf{E}}_{h}^{n+1}+\mathbf{G}^{n+1}\right\|_{0}\left\|\nabla \delta \widehat{\mathbf{E}}_{h}^{n+1}\right\|_{0} \\
\leq & \frac{\mu \tau}{6}\left\|\nabla \delta \widehat{\mathbf{E}}_{h}^{n+1}\right\|_{0}^{2}+\frac{C \tau}{\mu}\left(1+\frac{\tau}{h}\right)\left\|2 \delta \mathbf{E}^{n}-\delta \mathbf{E}^{n-1}\right\|_{0}^{2} \\
& +\frac{C \tau^{2}}{\mu}(\tau+h)^{2} \int_{t^{n-2}}^{t^{n+1}}\left\|\mathbf{u}_{t}(t)\right\|_{2}^{2} d t
\end{aligned}
$$


We now estimate $B_{2}$ using $\left\|2 \mathbf{E}^{n-1}-\mathbf{E}^{n-2}\right\|_{\mathbf{L}^{3}(\Omega)} \leq C h^{-d / 6}\left\|2 \mathbf{E}^{n-1}-\mathbf{E}^{n-2}\right\|_{0} \leq M$.

$$
\begin{aligned}
B_{2} \leq & C \tau\left\|2 \mathbf{E}^{n-1}-\mathbf{E}^{n-2}\right\|_{\mathbf{L}^{3}(\Omega)}\left\|\delta \mathbf{G}^{n+1}\right\|_{1}\left\|\nabla \delta \widehat{\mathbf{E}}_{h}^{n+1}\right\|_{0} \\
& +C \tau\left\|2 \mathbf{u}\left(t^{n-1}\right)-\mathbf{u}\left(t^{n-2}\right)\right\|_{1}\left\|\delta \mathbf{G}^{n+1}\right\|_{1}\left\|\nabla \delta \widehat{\mathbf{E}}_{h}^{n+1}\right\|_{0} \\
\leq & C \tau\left\|\delta \mathbf{G}^{n+1}\right\|_{1}^{2}+\frac{\mu \tau}{6}\left\|\nabla \delta \widehat{\mathbf{E}}_{h}^{n+1}\right\|_{0}^{2} \\
\leq & \frac{\mu \tau}{6}\left\|\nabla \delta \widehat{\mathbf{E}}_{h}^{n+1}\right\|_{0}^{2}+C \tau^{2} h^{2} \int_{t^{n-1}}^{t^{n+1}}\left(\left\|\mathbf{u}_{t}(t)\right\|_{2}^{2}+\left\|p_{t}(t)\right\|_{1}^{2}\right) d t .
\end{aligned}
$$

In light of Hölder inequality, (4.2) yields

$$
\begin{aligned}
A_{2} & =-2\left\langle 3 \delta \mathbf{G}^{n+1}-4 \delta \mathbf{G}^{n}+\delta \mathbf{G}^{n-1}, \delta \widehat{\mathbf{E}}_{h}^{n+1}\right\rangle \\
& \leq \frac{\mu \tau}{6}\left\|\nabla \delta \mathbf{E}_{h}^{n+1}\right\|_{0}^{2}+C h^{4} \int_{t^{n-2}}^{t^{n+1}}\left(\left\|\mathbf{u}_{t}(t)\right\|_{2}^{2}+\left\|p_{t}(t)\right\|_{1}^{2}\right) d t .
\end{aligned}
$$

Integral by parts leads

$$
\begin{aligned}
A_{3} & =4 \tau\left\langle\delta \delta P_{h}^{n+1}, \nabla \cdot \delta \widehat{\mathbf{E}}_{h}^{n+1}\right\rangle \\
& \leq \frac{\mu \tau}{6}\left\|\nabla \delta \widehat{\mathbf{E}}_{h}^{n+1}\right\|_{0}^{2}+\frac{C \tau^{4}}{\mu} \int_{t^{n-1}}^{t^{n+1}}\left(\left\|\mathbf{u}_{t t}(t)\right\|_{2}^{2}+\left\|p_{t t}(t)\right\|_{1}^{2}\right) d t .
\end{aligned}
$$

In order to tackle $A_{4}$, marking use of $\delta \widehat{\mathbf{E}}_{h}^{n+1}=\delta \mathbf{E}_{h}^{n+1}+\nabla \delta \delta \psi_{h}^{n+1}$. We readily get

$$
\begin{aligned}
& A_{4} \quad=-4 \tau\left\langle\nabla \delta \varepsilon_{h}^{n}, \delta \mathbf{E}_{h}^{n+1}+\nabla \delta \delta \psi_{h}^{n+1}\right\rangle \\
& =-\frac{8 \tau^{2}}{3}\left\langle\nabla \delta \varepsilon_{h}^{n}, \nabla\left(\delta \delta \varepsilon_{h}^{n+1}-\delta \delta P_{h}^{n+1}\right)\right\rangle \\
& \leq-\frac{4 \tau^{2}}{3}\left(\left\|\nabla \delta \varepsilon_{h}^{n+1}\right\|_{0}^{2}-\left\|\nabla \delta \varepsilon_{h}^{n}\right\|_{0}^{2}-\left\|\nabla \delta \delta \varepsilon_{h}^{n+1}\right\|_{0}^{2}\right) \\
& +C \tau^{3}\left\|\nabla \delta \varepsilon_{h}^{n}\right\|_{0}^{2}+C \tau\left\|\nabla \delta \delta P_{h}^{n+1}\right\|_{0}^{2} .
\end{aligned}
$$

If we now apply inequality $(a+b)^{2} \leq 4 a^{2}+\frac{4}{3} b^{2}$, then we can have

$$
\begin{aligned}
\frac{4 \tau^{2}}{3}\left\|\nabla \delta \delta \varepsilon_{h}^{n+1}\right\|_{0}^{2} & =\frac{4 \tau^{2}}{3}\left\|\nabla \delta \delta P_{h}^{n+1}+\frac{3}{2 \tau} \nabla \delta \delta \psi_{h}^{n+1}\right\|_{0}^{2} \\
& \leq C \tau^{2}\left\|\nabla \delta \delta P_{h}^{n+1}\right\|_{0}^{2}+4\left\|\nabla \delta \delta \psi_{h}^{n+1}\right\|_{0}^{2} .
\end{aligned}
$$

So we arrive at

$$
\begin{aligned}
A_{4} \leq & -\frac{4 \tau^{2}}{3}\left(\left\|\nabla \delta \varepsilon_{h}^{n+1}\right\|_{0}^{2}-\left\|\nabla \delta \varepsilon_{h}^{n}\right\|_{0}^{2}\right)+4\left\|\nabla \delta \delta \psi_{h}^{n+1}\right\|_{0}^{2} \\
& +C \tau^{3}\left\|\nabla \delta \varepsilon_{h}^{n}\right\|_{0}^{2}+C \tau^{4} \int_{t^{n-1}}^{t^{n+1}}\left(\left\|\mathbf{u}_{t t}(t)\right\|_{2}^{2}+\left\|p_{t t}(t)\right\|_{1}^{2}\right) d t .
\end{aligned}
$$


Invoking (4.7) and (2.11), (1.6) leads

$$
\begin{aligned}
A_{5} & =-4 \mu \tau\left\langle\delta q_{h}^{n}, \delta \delta q_{h}^{n+1}\right\rangle \\
& =-2 \mu \tau\left(\left\|\delta q_{h}^{n+1}\right\|_{0}^{2}-\left\|\delta q_{h}^{n}\right\|_{0}^{2}-\left\|\delta \delta q_{h}^{n+1}\right\|_{0}^{2}\right) \\
& \leq-2 \mu \tau\left(\left\|\delta q_{h}^{n+1}\right\|_{0}^{2}-\left\|\delta q_{h}^{n}\right\|_{0}^{2}\right)+2 \mu \tau\left\|\nabla \delta \widehat{\mathbf{E}}_{h}^{n+1}\right\|_{0}^{2} .
\end{aligned}
$$

Finally, $A_{6}$ term becomes

$$
\begin{aligned}
A_{6} & \leq C \tau\left\|\delta \mathbf{R}^{n+1}\right\|_{0}\left\|\delta \widehat{\mathbf{E}}_{h}^{n+1}\right\|_{0} \\
& \leq C \tau\left\|\delta \widehat{\mathbf{E}}_{h}^{n+1}\right\|_{0}^{2}+C \tau^{4} \int_{t^{n-1}}^{t^{n+1}}\left\|\mathbf{u}_{t t t}(t)\right\|_{0}^{2} d t .
\end{aligned}
$$

Inserting above estimates into (4.16) and summing for $n$ from 2 to $N$ yield

$$
\begin{aligned}
& \left\|\delta \mathbf{E}_{h}^{N+1}\right\|_{0}^{2}+\left\|2 \delta \mathbf{E}_{h}^{N+1}-\delta \mathbf{E}_{h}^{N}\right\|_{0}^{2}+\sum_{n=2}^{N}\left(\left\|\delta \delta \delta \mathbf{E}_{h}^{N+1}\right\|_{0}^{2}+\left\|\nabla \delta \delta \psi_{h}^{n+1}\right\|_{0}\right) \\
& +\mu \tau \sum_{n=2}^{N}\left\|\nabla \delta \widehat{\mathbf{E}}_{h}^{n+1}\right\|_{0}^{2}+\frac{4 \tau^{2}}{3}\left\|\nabla \delta \varepsilon_{h}^{N+1}\right\|_{0}^{2}+2 \mu \tau\left\|\delta q_{h}^{N+1}\right\|_{0}^{2} \leq\left\|\delta \mathbf{E}_{h}^{2}\right\|_{0}^{2} \\
& +\left\|2 \delta \mathbf{E}_{h}^{2}-\delta \mathbf{E}_{h}^{1}\right\|_{0}^{2}+\frac{4 \tau^{2}}{3}\left\|\nabla \delta \varepsilon_{h}^{2}\right\|_{0}^{2}+2 \mu \tau\left\|\delta q_{h}^{2}\right\|_{0}^{2}+C \tau^{2} \sum_{n=2}^{N}\left\|\nabla \delta \widehat{\mathbf{E}}_{h}^{n}\right\|_{0}^{2} \\
& +\frac{C \tau}{\mu}\left(1+\frac{\tau}{h}\right) \sum_{n=2}^{N}\left\|2 \delta \mathbf{E}_{h}^{n}-\delta \mathbf{E}_{h}^{n-1}\right\|_{0}^{2}+C \tau^{3} \sum_{n=2}^{N}\left\|\nabla \delta \varepsilon_{h}^{n}\right\|_{0}^{2} \\
& +C \frac{\left(\tau^{2}+h^{2}\right)^{2}}{\mu} \int_{0}^{t^{N+1}}\left(\left\|\mathbf{u}_{t t t}\right\|_{0}^{2}+\left\|\mathbf{u}_{t t}(t)\right\|_{2}^{2}+\left\|\mathbf{u}_{t}\right\|_{2}^{2}+\left\|p_{t t}(t)\right\|_{1}^{2}+\left\|p_{t}(t)\right\|_{1}^{2}\right) d t .
\end{aligned}
$$

We note here $\tau^{2} \sum_{n=2}^{N}\left\|\nabla \delta \widehat{\mathbf{E}}_{h}^{n}\right\|_{0}^{2}$ can be removed by cancellation with the term $\tau \sum_{n=2}^{N}\left\|\nabla \delta \widehat{\mathbf{E}}_{h}^{n}\right\|_{0}^{2}$ on the left hand side, provided $\tau$ is small enough. We need assumption $\tau=C h$ to impose $\frac{\tau}{h}=C$. If we apply Gronwall inequality and then use (4.14), then we arrive at (4.15) and complete the proof.

In order to extend to optimal accuracy, we use duality argument with the Stokes equations

$$
\begin{aligned}
-\Delta \mathbf{v}^{n+1}+\nabla r^{n+1} & =\mathbf{E}_{h}^{n+1}, \\
\nabla \cdot \mathbf{v}^{n+1} & =0, \quad \text { in } \Omega,
\end{aligned}
$$

with vanishing boundary condition $\mathbf{v}=\mathbf{0}$. And let $\left\{\mathbf{v}_{h}^{n+1}, r_{h}^{n+1}\right\} \in \mathbb{V}_{h} \times \mathbb{P}_{h}$ be the solution of the weak form of (4.17),

$$
\begin{aligned}
\left\langle\nabla \mathbf{v}_{h}^{n+1}, \nabla \mathbf{w}_{h}\right\rangle+\left\langle\nabla r_{h}^{n+1}, \mathbf{w}_{h}\right\rangle & =\left\langle\mathbf{E}_{h}^{n+1}, \mathbf{w}_{h}\right\rangle, & & \forall \mathbf{w}_{h} \in \mathbb{V}_{h} \\
\left\langle\nabla \cdot \mathbf{v}_{h}^{n+1}, \phi_{h}\right\rangle & =0, & & \forall \phi_{h} \in \mathbb{P}_{h} .
\end{aligned}
$$


According to Assumption 1, we have

$$
\left\|\mathbf{v}^{n+1}\right\|_{2}+\left\|r^{n+1}\right\|_{1} \leq\left\|\mathbf{E}^{n+1}\right\|_{0}
$$

and so Lemma 2.3 yields

$$
\begin{aligned}
\left\|\mathbf{v}^{n+1}-\mathbf{v}_{h}^{n+1}\right\|_{0}+h\left\|\mathbf{v}^{n+1}-\mathbf{v}_{h}^{n+1}\right\|_{1}+h\left\|r^{n+1}-r_{h}^{n+1}\right\|_{0} & \leq h^{2}\left\|\mathbf{E}^{n+1}\right\|_{0}, \\
\left\|\mathbf{v}^{n+1}-\mathbf{v}_{h}^{n+1}\right\| & \leq\left\|\mathbf{E}^{n+1}\right\|_{0} .
\end{aligned}
$$

In order to use in proof of next lemma, we derive 2 equations by considering for the case $\mathbf{w}_{h}=\mathbf{v}_{h}^{n+1}$ in (4.18)

$$
\begin{array}{r}
\left\langle 3 \widehat{\mathbf{E}}^{n+1}-4 \mathbf{E}^{n}+\mathbf{E}^{n-1}, \mathbf{v}_{h}^{n+1}\right\rangle=\left\langle 3 \mathbf{G}^{n+1}-4 \mathbf{G}^{n}+\mathbf{G}^{n-1}, \mathbf{v}_{h}^{n+1}\right\rangle \\
+\left\langle 3 \nabla \mathbf{v}_{h}^{n+1}-4 \nabla \mathbf{v}_{h}^{n}+\nabla \mathbf{v}_{h}^{n-1}, \nabla \mathbf{v}_{h}^{n+1}\right\rangle
\end{array}
$$

and by choosing $\mathbf{w}_{h}=\widehat{\mathbf{E}}_{h}^{n+1}$ in $(4.18)$

$$
\left\langle\nabla \widehat{\mathbf{E}}_{h}^{n+1}, \nabla \mathbf{v}_{h}^{n+1}\right\rangle=\left\langle\widehat{\mathbf{E}}_{h}^{n+1}, \mathbf{E}_{h}^{n+1}\right\rangle-\left\langle\nabla r_{h}^{n+1}, \widehat{\mathbf{E}}_{h}^{n+1}\right\rangle .
$$

LEMMA 4.4 (Full rate of convergence for velocity). Let the pairs $\left(\mathbf{v}^{n+1}\right.$, $\left.r^{n+1}\right)$ and $\left(\mathbf{v}_{h}^{n+1}, r_{h}^{n+1}\right)$ be the solutions of (4.17) and (4.18), respectively. Let the exact solution of (1.1) is smooth enough and $\tau=C h$. If Assumptions 1-2 and 4-5 hold, then we have

$$
\begin{array}{r}
\left\|\nabla \mathbf{v}_{h}^{N+1}\right\|_{0}^{2}+\left\|\nabla\left(2 \mathbf{v}_{h}^{N+1}-\mathbf{v}_{h}^{N}\right)\right\|_{0}^{2}+\sum_{n=1}^{N}\left\|\nabla \delta \delta \mathbf{v}_{h}^{n+1}\right\|_{0}^{2} \\
+2 \mu \tau \sum_{n=1}^{N}\left\|\mathbf{E}_{h}^{n+1}\right\|_{0}^{2} \leq C\left(\tau^{4}+h^{4}\right) .
\end{array}
$$

Proof. We choose $\mathbf{w}_{h}=4 \tau \mathbf{v}_{h}^{n+1} \in \mathbb{V}_{h}$ in (4.11) and then we apply (4.21) and (4.22) to obtain

$$
\begin{aligned}
\left\|\nabla \mathbf{v}_{h}^{n+1}\right\|_{0}^{2}+ & \left\|\nabla\left(2 \mathbf{v}_{h}^{n+1}-\mathbf{v}_{h}^{n}\right)\right\|_{0}^{2}+\left\|\nabla \delta \delta \mathbf{v}_{h}^{n+1}\right\|_{0}^{2}-\left\|\nabla \mathbf{v}_{h}^{n}\right\|_{0}^{2} \\
& -\left\|\nabla\left(2 \mathbf{v}_{h}^{n}-\mathbf{v}_{h}^{n-1}\right)\right\|_{0}^{2}+4 \mu \tau\left\|\mathbf{E}_{h}^{n+1}\right\|_{0}^{2}=\sum_{i=1}^{4} A_{i},
\end{aligned}
$$

where

$$
\begin{aligned}
& A_{1}:=4 \tau \mathcal{N}\left(2 \mathbf{u}_{h}^{n}-\mathbf{u}_{h}^{n-1}, \widehat{\mathbf{u}}_{h}^{n+1}, \mathbf{v}_{h}^{n+1}\right)-4 \tau \mathcal{N}\left(\mathbf{u}\left(t^{n+1}\right), \mathbf{u}\left(t^{n+1}\right), \mathbf{v}_{h}^{n+1}\right), \\
& A_{2}:=-2\left\langle 3 \mathbf{G}^{n+1}-4 \mathbf{G}^{n}+\mathbf{G}^{n-1}, \mathbf{v}_{h}^{n+1}\right\rangle, \\
& A_{4}:=4 \tau\left\langle\mathbf{R}^{n+1}, \mathbf{v}_{h}^{n+1}\right\rangle,
\end{aligned}
$$


We now estimate $A_{1}$ to $A_{4}$ separately. The convection term $A_{1}$ can be rewritten as follows:

$$
\begin{aligned}
A_{1}= & -4 \tau \mathcal{N}\left(\delta \delta \mathbf{u}\left(t^{n+1}\right), \mathbf{u}\left(t^{n+1}\right), \mathbf{v}_{h}^{n+1}\right)+4 \tau \mathcal{N}\left(2 \mathbf{E}^{n}-\mathbf{E}^{n-1}, \widehat{\mathbf{E}}^{n+1}, \mathbf{v}_{h}^{n+1}\right) \\
& -4 \tau \mathcal{N}\left(2 \mathbf{u}\left(t^{n}\right)-\mathbf{u}\left(t^{n-1}\right), \widehat{\mathbf{E}}^{n+1}, \mathbf{v}_{h}^{n+1}\right) \\
& -4 \tau \mathcal{N}\left(2 \mathbf{E}^{n}-\mathbf{E}^{n-1}, \mathbf{u}\left(t^{n+1}\right), \mathbf{v}_{h}^{n+1}\right)=\sum_{i=1}^{4} A_{1, i} .
\end{aligned}
$$

To estimate convection terms, we will use frequently Lemma 2.5 without notice. Using $\left\|\mathbf{u}\left(t^{n+1}\right)\right\|_{2} \leq M$, we can readily get

$$
\begin{aligned}
A_{1,1} & \leq C \tau\left\|\delta \delta \mathbf{u}\left(t^{n+1}\right)\right\|_{0}\left\|\mathbf{u}\left(t^{n+1}\right)\right\|_{2}\left\|\mathbf{v}_{h}^{n+1}\right\|_{1} \\
& \leq C \tau\left\|\nabla \mathbf{v}_{h}^{n+1}\right\|_{0}^{2}+C \mu \tau^{4} \int_{t^{n-1}}^{t^{n+1}}\left\|\mathbf{u}_{t t}(t)\right\|_{0}^{2} d t
\end{aligned}
$$

and

$$
\begin{aligned}
A_{1,4} & \leq C \tau\left(\left\|\mathbf{E}^{n}\right\|_{0}+\left\|\delta \mathbf{E}^{n}\right\|_{0}\right)\left\|\mathbf{u}\left(t^{n+1}\right)\right\|_{2}\left\|\mathbf{v}_{h}^{n+1}\right\|_{1} \\
& \leq \frac{\mu \tau}{2}\left(\left\|\mathbf{E}_{h}^{n}\right\|_{0}^{2}+\left\|\mathbf{G}^{n}\right\|_{0}^{2}+\left\|\delta \mathbf{E}_{h}^{n}\right\|_{0}^{2}+\left\|\delta \mathbf{G}^{n}\right\|_{0}^{2}\right)+\frac{C \tau}{\mu}\left\|\nabla \mathbf{v}_{h}^{n+1}\right\|_{0}^{2} .
\end{aligned}
$$

Because $\nabla \cdot\left(2 \mathbf{u}\left(t^{n}\right)-\mathbf{u}\left(t^{n-1}\right)\right)=0$ and $2 \mathbf{u}\left(t^{n}\right)-\mathbf{u}\left(t^{n-1}\right)=\mathbf{0}$ on boundary, we can use (2.6) and so we get

$$
\begin{aligned}
A_{1,3} & \leq C \tau\left\|2 \mathbf{u}\left(t^{n}\right)-\mathbf{u}\left(t^{n-1}\right)\right\|_{2}\left\|\widehat{\mathbf{E}}^{n+1}\right\|\left\|_{0}\right\| \mathbf{v}_{h}^{n+1} \|_{1} \\
& \leq \frac{\mu \tau}{2}\left(\left\|\mathbf{E}_{h}^{n+1}\right\|_{0}^{2}+\left\|\mathbf{G}^{n+1}\right\|_{0}^{2}+\left\|\nabla \delta \psi_{h}^{n+1}\right\|_{0}^{2}\right)+\frac{C \tau}{\mu}\left\|\nabla \mathbf{v}_{h}^{n+1}\right\|_{0}^{2} .
\end{aligned}
$$

We now apply

$$
\begin{aligned}
\left\|\nabla \delta \psi_{h}^{n+1}\right\|_{0}^{2} & =\frac{4 \tau^{2}}{9}\left\|\nabla\left(\delta \varepsilon_{h}^{n+1}-\delta P_{h}^{n+1}\right)\right\|_{0}^{2} \\
& \leq C \tau^{2}\left\|\nabla \delta \varepsilon_{h}^{n+1}\right\|_{0}^{2}+C \tau^{3} \int_{t^{n}}^{t^{n+1}}\left(\left\|\mathbf{u}_{t}(t)\right\|_{2}^{2}+\left\|p_{t}(t)\right\|_{1}^{2}\right) d t
\end{aligned}
$$

to derive

$$
\begin{aligned}
A_{1,3} \leq & \frac{\mu \tau}{2}\left(\left\|\mathbf{E}_{h}^{n+1}\right\|_{0}^{2}+\left\|\mathbf{G}^{n+1}\right\|_{0}^{2}\right)+C \mu \tau^{3}\left\|\nabla \delta \varepsilon_{h}^{n+1}\right\|_{0}^{2} \\
& +C \mu \tau^{4} \int_{t^{n}}^{t^{n+1}}\left(\left\|\mathbf{u}_{t}(t)\right\|_{2}^{2}+\left\|p_{t}(t)\right\|_{1}^{2}\right) d t+\frac{C \tau}{\mu}\left\|\nabla \mathbf{v}_{h}^{n+1}\right\|_{0}^{2} .
\end{aligned}
$$

If we apply $\left\|2 \mathbf{E}^{n}-\mathbf{E}^{n-1}\right\|_{\mathbf{L}^{3}(\Omega)} \leq C h^{-d / 6}\left\|2 \mathbf{E}^{n}-\mathbf{E}^{n-1}\right\|_{0} \leq C h^{-d / 6}(\tau+h)$ which derives from Lemmas 2.1 and 4.1, then we can derive, by using Lemma 2.5 and 
(4.19)-(4.20),

$$
\begin{aligned}
A_{1,2} \leq & C \tau\left\|2 \mathbf{E}^{n}-\mathbf{E}^{n-1}\right\|_{0}\left\|\widehat{\mathbf{E}}^{n+1}\right\|_{1}\left\|\mathbf{v}^{n+1}\right\|_{2} \\
& +C \tau\left\|2 \mathbf{E}^{n}-\mathbf{E}^{n-1}\right\|_{\mathbf{L}^{3}(\Omega)}\left\|\widehat{\mathbf{E}}^{n+1}\right\|_{1}\left\|\mathbf{v}^{n+1}-\mathbf{v}_{h}^{n+1}\right\|_{1} \\
\leq & C \tau(\tau+h)\left\|\widehat{\mathbf{E}}^{n+1}\right\|_{1}\left\|\mathbf{v}^{n+1}\right\|_{2}+C \tau h\left\|\widehat{\mathbf{E}}^{n+1}\right\|_{1}\left\|\mathbf{v}^{n+1}\right\|_{2} \\
\leq & \frac{C}{\mu} \tau(\tau+h)^{2}\left(\left\|\nabla \widehat{\mathbf{E}}_{h}^{n+1}\right\|_{0}^{2}+\left\|\nabla \mathbf{G}^{n+1}\right\|_{0}^{2}\right)+\frac{\mu \tau}{2}\left\|\mathbf{E}_{h}^{n+1}\right\|_{0}^{2} .
\end{aligned}
$$

In conjunction with (4.2), we can have

$$
\begin{aligned}
A_{2} & \leq \frac{C}{\tau}\left(\left\|\delta \mathbf{G}^{n+1}\right\|_{0}^{2}+\left\|\delta \mathbf{G}^{n}\right\|_{0}^{2}\right)+C \tau\left\|\mathbf{v}_{h}^{n+1}\right\|_{0}^{2} \\
& \leq C \tau\left\|\mathbf{v}_{h}^{n+1}\right\|_{0}^{2}+C h^{4} \int_{t^{n-1}}^{t^{n+1}}\left(\left\|\mathbf{u}_{t}(t)\right\|_{2}^{2}+\left\|p_{t}(t)\right\|_{1}^{2}\right) d t .
\end{aligned}
$$

The definition of $\widehat{\mathbf{E}}_{h}^{n+1}=\mathbf{E}_{h}^{n+1}+\nabla \delta \psi_{h}^{n+1}$ and (4.19) gives us

$$
A_{3}=4 \mu \tau\left\langle\nabla \delta \psi_{h}^{n+1}, \nabla r_{h}^{n+1}\right\rangle \leq \mu \tau\left\|\mathbf{E}_{h}^{n+1}\right\|_{0}^{2}+\frac{C \tau}{\mu}\left\|\nabla \delta \psi_{h}^{n+1}\right\|_{0}^{2} .
$$

If we apply (4.25) again, then we arrive at

$$
A_{3} \leq \mu \tau\left\|\mathbf{E}_{h}^{n+1}\right\|_{0}^{2}+\frac{C \tau^{3}}{\mu}\left\|\nabla \delta \varepsilon_{h}^{n+1}\right\|_{0}^{2}+\frac{C \tau^{4}}{\mu} \int_{t^{n}}^{t^{n+1}}\left\|\nabla p_{t}(t)\right\|_{0}^{2} d t .
$$

On the other hand, the truncation error term becomes

$$
A_{4}=4 \tau\left\langle\mathbf{R}^{n+1}, \mathbf{v}_{h}^{n+1}\right\rangle \leq C \tau\left\|\nabla \mathbf{v}_{h}^{n+1}\right\|_{0}^{2}+C \tau^{4} \int_{t^{n-1}}^{t^{n+1}}\left\|\mathbf{u}_{t t t}(s)\right\|_{0}^{2} d t .
$$

Invoking $\mathbf{v}^{0}=\mathbf{0}$, inserting above estimates from $A_{1}$ and $A_{4}$ into (4.24) and summing over $n$ from 1 to $N$ give us

$$
\begin{aligned}
& \left\|\nabla \mathbf{v}_{h}^{N+1}\right\|_{0}^{2}+\left\|\nabla\left(2 \mathbf{v}_{h}^{N+1}-\mathbf{v}_{h}^{N}\right)\right\|_{0}^{2}+\sum_{n=1}^{N}\left\|\nabla \delta \delta \mathbf{v}_{h}^{n+1}\right\|_{0}^{2}+\mu \tau \sum_{n=1}^{N}\left\|\mathbf{E}_{h}^{n+1}\right\|_{0}^{2} \\
& \leq 5\left\|\nabla \mathbf{v}_{h}^{1}\right\|_{0}^{2}+C \tau(\tau+h)^{2} \sum_{n=1}^{N}\left(\left\|\nabla \widehat{\mathbf{E}}_{h}^{n+1}\right\|_{0}^{2}+\left\|\nabla \mathbf{G}^{n+1}\right\|_{0}^{2}+\left\|\nabla \delta \varepsilon_{h}^{n+1}\right\|_{0}^{2}\right) \\
& +C \tau \sum_{n=1}^{N}\left\|\nabla \mathbf{v}_{h}^{n+1}\right\|_{0}^{2} \\
& +C \mu \tau \sum_{n=1}^{N}\left(\left\|\mathbf{E}_{h}^{n+1}\right\|_{0}^{2}+\left\|\delta \mathbf{E}_{h}^{n+1}\right\|_{0}^{2}+\left\|\mathbf{G}^{n+1}\right\|_{0}^{2}+\left\|\delta \mathbf{G}^{n}\right\|_{0}^{2}\right) \\
& +C \mu\left(\tau^{4}+h^{4}\right) \int_{0}^{t^{N+1}}\left(\left\|\mathbf{u}_{t t t}(t)\right\|_{0}^{2}+\left\|\mathbf{u}_{t t}(t)\right\|_{0}^{2}+\left\|\mathbf{u}_{t}(t)\right\|_{2}^{2}+\left\|p_{t}(t)\right\|_{1}^{2}\right) d t .
\end{aligned}
$$


Applying the discrete Gronwall inequality and Lemmas 4.1 and 4.3, we obtain (4.23).

We now estimate the pressure error in $L^{2}\left(0, T ; L^{2}(\Omega)\right)$. This hinges on the error estimate for the time derivative of velocity of Lemma 4.3 .

LEMMA 4.5 (Pressure error estimate). Let the exact solution of (1.1) is smooth enough and $\tau=C h$. If Assumptions 1-5 hold, then we have

$$
\tau \sum_{n=1}^{N}\left\|e_{h}^{n+1}\right\|_{0}^{2} \leq C\left(\tau^{2}+h^{2}\right) .
$$

ProOF. We first recall again inf-sup condition in Assumption 3. Consequently, it suffices to estimate $\left\langle e^{n+1}, \nabla \cdot \mathbf{w}\right\rangle$ in terms of $\|\nabla \mathbf{w}\|_{0}$. In conjunction with (1.7), we can rewrite $(4.11)$ as

$$
\begin{aligned}
& \left\langle e_{h}^{n+1}, \nabla \cdot \mathbf{w}_{h}\right\rangle=\frac{1}{2 \tau}\left\langle 3 \mathbf{E}^{n+1}-4 \mathbf{E}^{n}+\mathbf{E}^{n-1}, \mathbf{w}_{h}\right\rangle \\
& +\mu\left\langle\nabla \widehat{\mathbf{E}}_{h}^{n+1}, \nabla \mathbf{w}_{h}\right\rangle+\mathcal{N}\left(\delta \delta \mathbf{u}\left(t^{n+1}\right), \mathbf{u}\left(t^{n+1}\right), \mathbf{w}_{h}\right) \\
& +\mathcal{N}\left(2 \mathbf{u}\left(t^{n+1}\right)-\mathbf{u}\left(t^{n}\right), \widehat{\mathbf{E}}^{n+1}, \mathbf{w}_{h}\right)+\mathcal{N}\left(2 \mathbf{E}^{n}-\mathbf{E}^{n-1}, \widehat{\mathbf{u}}_{h}^{n+1}, \mathbf{w}_{h}\right) \\
& -\mu\left\langle\nabla \delta q_{h}^{n+1}, \mathbf{w}_{h}\right\rangle-\left\langle\mathbf{R}^{n+1}, \mathbf{w}_{h}\right\rangle=\sum_{i=1}^{7} A_{i} .
\end{aligned}
$$

We now proceed to estimate each term $A_{1}$ to $A_{7}$ separately. We readily obtain

$$
A_{1} \leq \frac{C}{\tau}\left(\left\|\delta \mathbf{E}^{n+1}\right\|_{0}+\left\|\delta \mathbf{E}^{n}\right\|_{0}\right)\left\|\mathbf{w}_{h}\right\|_{0} \leq \frac{C}{\tau}\left(\left\|\delta \mathbf{E}^{n+1}\right\|_{0}+\left\|\delta \mathbf{E}^{n}\right\|_{0}\right)\left\|\nabla \mathbf{w}_{h}\right\|_{0}
$$

and

$$
A_{2} \leq C\left\|\nabla \widehat{\mathbf{E}}_{h}^{n+1}\right\|_{0}\left\|\nabla \mathbf{w}_{h}\right\|_{0} .
$$

Term $A_{3}$ and $A_{4}$ can be dealt with the aid of Lemma 2.5 and $\left\|\mathbf{u}\left(t^{n+1}\right)\right\|_{2} \leq M$ as follows:

$$
A_{3} \leq C\left\|\delta \delta \mathbf{u}\left(t^{n+1}\right)\right\|_{0}\left\|\mathbf{u}\left(t^{n+1}\right)\right\|_{2}\left\|\mathbf{w}_{h}\right\|_{1} \leq C\left\|\delta \delta \mathbf{u}\left(t^{n+1}\right)\right\|_{1}\left\|\nabla \mathbf{w}_{h}\right\|_{1}
$$

and

$$
A_{4} \leq C\left\|2 \mathbf{u}\left(t^{n+1}\right)-\mathbf{u}\left(t^{n}\right)\right\|_{2}\left\|\widehat{\mathbf{E}}^{n+1}\right\|_{0}\left\|\mathbf{w}_{h}\right\|_{1} \leq C\left\|\widehat{\mathbf{E}}^{n+1}\right\|_{0}\left\|\nabla \mathbf{w}_{h}\right\|_{0} .
$$

In light of $\left\|\widehat{\mathbf{u}}_{h}^{n+1}\right\|_{1}=\left\|\widehat{\mathbf{E}}^{n+1}-\mathbf{u}\left(t^{n+1}\right)\right\|_{1} \leq C$ from Lemma 4.1, we can have

$$
\begin{aligned}
A_{5} & \leq C\left\|2 \mathbf{E}^{n}-\mathbf{E}^{n-1}\right\|_{\mathbf{L}^{3}(\Omega)}\left\|\widehat{\mathbf{u}}_{h}^{n+1}\right\|_{1}\left\|\mathbf{w}_{h}\right\|_{1} \\
& \leq \frac{C}{\sqrt{h}}\left(\left\|\mathbf{E}^{n+1}\right\|_{0}+\left\|\mathbf{E}^{n}\right\|_{0}\right)\left\|\nabla \mathbf{w}_{h}\right\|_{0} .
\end{aligned}
$$

Integrate by parts and Hölder inequality yield

$$
A_{6} \leq C\left\|\delta q_{h}^{n+1}\right\|_{0}\left\|\nabla \mathbf{w}_{h}\right\|_{0} .
$$

On the other hand, we have

$$
A_{7} \leq\left\|\mathbf{R}^{n+1}\right\|_{-1}\|\nabla \mathbf{w}\|_{0}
$$


Inserting the estimates for $A_{1}$ to $A_{7}$ back into (4.27), and employing discrete inf-sup condition in Assumption 3, we obtain

$$
\begin{aligned}
C\left\|e^{n+1}\right\|_{0} \leq & \frac{1}{\tau}\left(\left\|\delta \mathbf{E}^{n+1}\right\|_{0}+\left\|\delta \mathbf{E}^{n}\right\|_{0}\right)+\frac{C}{\sqrt{h}}\left(\left\|\mathbf{E}^{n+1}\right\|_{0}+\left\|\mathbf{E}^{n}\right\|_{0}\right)+\left\|\delta q_{h}^{n+1}\right\|_{0} \\
& +\left\|\nabla \widehat{\mathbf{E}}_{h}^{n+1}\right\|_{0}+\left\|\delta \delta \mathbf{u}\left(t^{n+1}\right)\right\|_{1}+\left\|\widehat{\mathbf{E}}^{n+1}\right\|_{0}+\left\|\mathbf{R}^{n+1}\right\|_{-1} .
\end{aligned}
$$

If we now square, multiply by $\tau$, and sum over $n$ from 1 to $N$, then Lemmas 4.1 and 4.3-4.4 derives (4.26).

\section{Numerical experiments}

In this section, we perform two numerical experiments: the first is to check accuracy and the second is to test stability. In the first experiments, we choose square domain $[0,1] \times[0,1]$ and impose forcing term the exact solution to become

$$
\begin{aligned}
& u=\exp (t) \sin ^{2}(\pi x) \sin (2 \pi y), \\
& v=-\exp (t) \sin (2 \pi x) \sin ^{2}(\pi y), \\
& p=\exp (t) \cos (\pi x) \cos (\pi y) .
\end{aligned}
$$

\begin{tabular}{|c||c|c|c|c|c|}
\hline$\tau=h$ & $1 / 16$ & $1 / 32$ & $1 / 64$ & $1 / 128$ & $1 / 256$ \\
\hline \hline \multirow{2}{*}{$\|E\|_{0}$} & 0.00384017 & 0.00130831 & 0.000391824 & 0.000107996 & $2.8413 \mathrm{e}-05$ \\
\cline { 2 - 6 } & Order & 1.553466 & 1.739427 & 1.859228 & 1.926355 \\
\hline \multirow{2}{*}{$\|E\|_{L^{\infty}}$} & 0.0112291 & 0.00381103 & 0.0011382 & 0.00031345 & $8.24393 \mathrm{e}-05$ \\
\cline { 2 - 6 } & Order & 1.558989 & 1.743427 & 1.860447 & 1.926831 \\
\hline \multirow{2}{*}{$\|E\|_{1}$} & 0.0798332 & 0.0239901 & 0.00680859 & 0.00183099 & 0.000476245 \\
\cline { 2 - 6 } & Order & 1.734550 & 1.817011 & 1.894732 & 1.942848 \\
\hline \multirow{2}{*}{$\|e\|_{0}$} & 0.0986215 & 0.0332739 & 0.0099548 & 0.00267395 & 0.000704462 \\
\cline { 2 - 6 } & Order & 1.567511 & 1.740927 & 1.896420 & 1.924379 \\
\hline \multirow{2}{*}{$\|e\|_{L^{\infty}}$} & 0.53446 & 0.214847 & 0.0759942 & 0.0236211 & 0.0070079 \\
\cline { 2 - 6 } & Order & 1.314772 & 1.499348 & 1.685813 & 1.753022 \\
\hline
\end{tabular}

TABLE 1. Error decay for Algorithm 1

Table 1 is the error decay for Algorithm 1. In this computation, we use TaylorHood (P2-P1) finite element on the uniform mesh. We impose $\tau=h$ and $\mu=1$. These error decay is optimal and consists to Theorem 2.

In [9], a rectangular driven cavity of low viscosity flow is performed in a domain $[0,0.75] \times[0,1]$ with initial and boundary conditions as described in Figure 1 . In this paper, we carry out the experiment with $\mu=1 / 10,000$ and $h=1 / 256$, and then we hire $\tau=0.5$ as big as possible. Most of numerical algorithms have upper bound for 
the size of $\tau$ to make hold the stability constraint, like $\tau \leq C h$, where $C$ depends on the Reynolds numbers. So, the smaller $\tau$ has to be imposed for the bigger Reynolds numbers problem. But Algorithm 1 becomes released from the limitation of the time marching size $\tau$ by Theorem 1 .

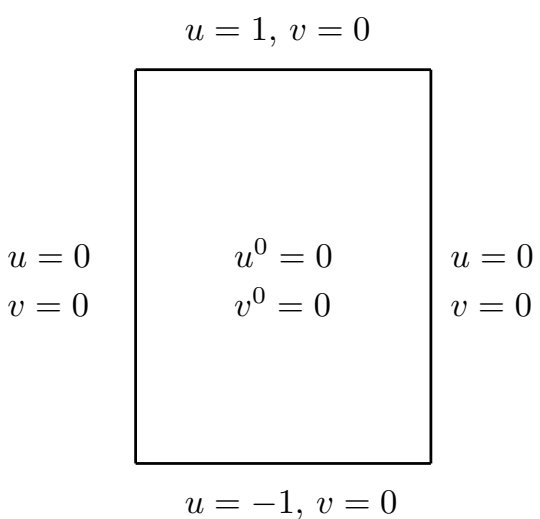

FigURE 1. Initial and boundary conditions for rectangular driven cavity flow in the domain $[0,0.75] \times[0,1]$.

Figure 2 is the numerical result of the rectangular driven cavity flow at time $T=100$ of Algorithm 1 and displays still stable even for high viscosity flow with $\mu=1 / 10,000$ under extremely strong unstable conditions $h=1 / 256$ and $\tau=0.5$. The $\tau=0.5$ is extremely big size for this case, but it is still stable. We thus can conclude that Algorithm 1 is unconditionally stable and consists to Theorem 1 . We note here that this experiment is to verify only stability for any $\tau$, not to check accuracy. So we impose very big $\tau=0.5$ and the big $\tau$ is the main reason of the oscillations in Figure 2. We need to use a reasonable $\tau$, if we want to obtain more accurate results, because stability and accuracy do not depend each other.

\section{References}

[1] S.C. Brenner and L.R. Scott, The Mathematical Theory of Finite Element Methods Springer-Verlag, (1994).

[2] F. Brezzi and M. Fortin, Mixed and Hybrid Finite Element Methods, SpringerVerlag, (1991).

[3] A.J. Chorin, Numerical solution of the Navier-Stokes equations, Math. Comp., 22 (1968), 745-762.

[4] P. Constantin and C. Foias, Navier-Stokes Equations, The University of Chicago Press (1988).

[5] V. Girault, and P.A. Raviart, Finite Element Methods for Navier-Stokes Equations, Springer-Verlag (1986). 


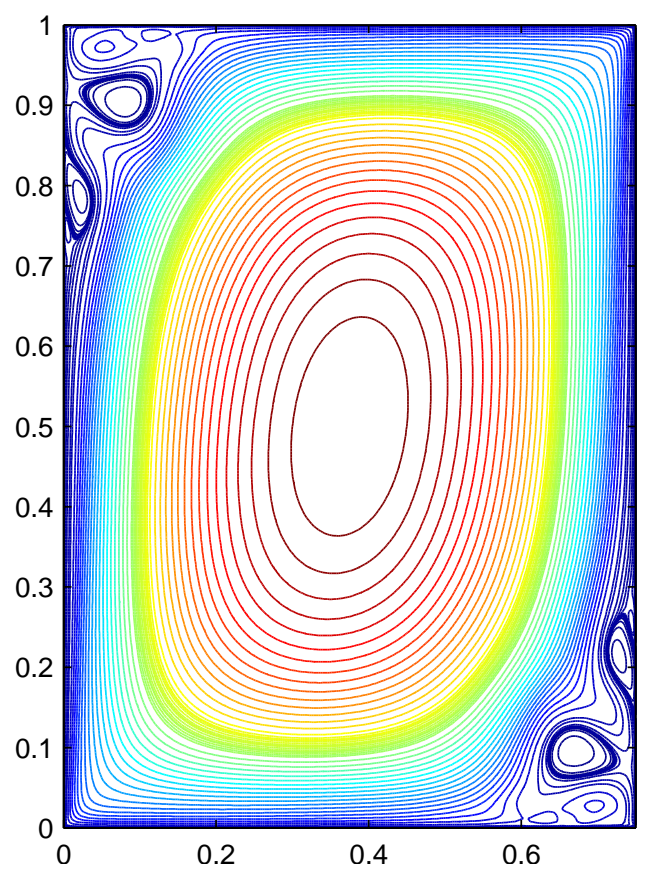

FIGURE 2. Rectangular driven cavity streamline at $T=$ 100 for Algorithm 1 with $\mu=1 / 10,000, h=1 / 256, \tau=$ 0.5

[6] J.L. Guermond and J. Shen. A new class of truly consistent splitting schemes for incompressible flows. J. Comput. Phys., 192:262-276, 2003.

[7] J.L. Guermond and J. Shen On the error estimates of rotational pressurecorrection projection methods, Math. Comp., 73 (2004), 1719-1737.

[8] J.G. Heywood and R. Rannacher, Finite element approximation of the nonstationary Navier-stokes problem. I. regularity of solutions and second-order error estimates for spatial discretization, SIAM J. Numer. Anal., 19 (1982), 275311.

[9] K. Ito and Z. Qiao A high order compact MAC finite difference scheme for the Stokes equations: augmented variable approach, J. Comput. Phys., 227 (2008), $8177-8190$.

[10] R.B. Kellogg and J.E. Osborn, A regularity result for the stokes problems in a convex polygon, J. Funct. Anal., 21 (1976), 397-431.

[11] R.H. Nochetto and J.-H. Pyo, A finite element Gauge-Uzawa method. Part I : the Navier-Stokes equations, SIAM J. Numer. Anal., 43 (2005), 1043-1068.

[12] R.H. Nochetto and J.-H. Pyo, A finite element Gauge-Uzawa method. Part II : Boussinesq equations, Math. Models Methods Appl. Sci., 16 (2006), 1599-1626. 
[13] A. Prohl, Projection and Quasi-Compressiblity Methods for Solving the Incompressible Navier-Stokes Equations, B.G.Teubner Stuttgart (1997).

[14] J.-H. Pyo Error estimates for the second order semi-discrete stabilized GaugeUzawa method For the Navier-Stokes equations, Inter. J. Numer. Anal. and Model, 10 (2013), 24-41.

[15] J.-H. Pyo and J. Shen, Normal mode analysis of second-order projection methods for incompressible flows, Discrete Contin. Dyn. Syst. Ser. B, 5 (2005), 817-840.

[16] J.-H. Pyo and J. Shen, Gauge Uzawa methods for incompressible flows with Variable Density, J. Comput. Phys., 211 (2007), 181-197.

[17] R. Temam, Sur l'approximation de la solution des equations de Navier-Stokes par la methode des pas fractionnaires. II, Arch. Rational Mech. Anal., 33 (1969), 377-385.

[18] R. Temam, Navier-Stokes Equations, AMS Chelsea Publishing, (2001).

[19] L.J.P. Timmermanns, P.D. Minev, and F.N. Van De Vosse, An approximate projection scheme for incompressible flow using spectral elements, Int. J. Num. Meth. Fluids, 22 (1996), 673-688.

Department of Mathematics

Kangwon National University

200-701, Chuncheon

Republic of Korea

URL: http://math.kangwon.ac. kr/ jhpyo/

E-mail: jhpyo@kangwon.ac.kr 